\title{
THE CAUCHY PROBLEM FOR FRACTIONAL CAMASSA-HOLM EQUATION IN BESOV SPACE
}

\author{
LILI FAN, HONGJUN GAO, JUNFANG WANG, AND WEI YAN ${ }^{\dagger}$
}

\begin{abstract}
In this paper, we consider the fractional Camassa-Holm equation modelling the propagation of small-but-finite amplitude long unidirectional waves in a nonlocally and nonlinearly elastic medium. First, we establish the local well-posedness in Besov space $B_{2,1}^{s_{0}}$ with $s_{0}=2 \nu-\frac{1}{2}$ for $\nu>\frac{3}{2}$ and $s_{0}=\frac{5}{2}$ for $1<\nu \leq \frac{3}{2}$. Then, with a given analytic initial data, we establish the analyticity of the solutions in both variables, globally in space and locally in time.
\end{abstract}

Keywords: Besov spaces; Fractional Camassa-Holm equation; Local well-posedness; Blow-up criterion; Analyticity

AMS Subject Classification (2010): 35Q53; 35B30; 35G25.

\section{INTRODUCTION}

This paper is concerned with an evolution equation, named fractional Camassa-Holm (fCH) equation, which models the propagation of small-but-finite amplitude, long unidirectional waves in a one-dimensional infinite, homogeneous medium made of nonlocally and nonlinearly elastic material [18]

$$
\begin{aligned}
u_{t} & +u_{x}+u u_{x}+\frac{3}{4}\left(-\partial_{x}^{2}\right)^{\nu} u_{x}+\frac{5}{4}\left(-\partial_{x}^{2}\right)^{\nu} u_{t} \\
& +\frac{1}{4}\left[2\left(-\partial_{x}^{2}\right)^{\nu}\left(u u_{x}\right)+u\left(-\partial_{x}^{2}\right)^{\nu} u_{x}\right]=0, \quad x \in \mathbb{R}, \quad t>0,
\end{aligned}
$$

where $\nu \geq 1$ is a constant which may not be an integer. It is remarkable that when $\nu=1$, (1.1) reduces to the following classical Camassa-Holm $(\mathrm{CH})$ equation

$$
u_{t}+k_{1}\left(u_{x}-u_{x x x}\right)+3 u u_{x}-u_{x x t}=k_{2}\left(2 u_{x} u_{x x}+u u_{x x x}\right) .
$$

This prominent $\mathrm{CH}$ equation, for which the ratio of the nonlinear terms of (1.2) being $3: 2: 1$, was first derived formally by Fuchssteiner and Fokas [22] as a bi-Hamiltonian equation and later derived in the context of water waves as a model for unidirectional propagation of shallow water waves of moderate amplitude by Camassa and Holm [5] (see also the alternative derivation in [8, 14, 30]). The $\mathrm{CH}$ equation has been studied extensively in the last twenty years because of its many remarkable properties: infinitely many conservation laws and complete integrability [5, 19], existence of peaked solitons and multi-peakons [1, 5], well-posedness and breaking waves [3, 4, 9, 11, 12, 13, 15, 16, 27], just to mention a few. 
Recent years, increasing attention has been paid to the fractional equations. For instance, the fractional Korteweg-de Vries (fKdV) equation and the fractional BenjaminBona-Mahony (fBBM) equation have been obtained and studied in [18, 29, 37], the Camassa-Holm equations with fractional dissipation and the Camassa-Holm equations with fractional laplacian viscosity have been investigated in [24, 25]. Concerning the fCH equation (1.1), the local well-posedness for initial data $u_{0} \in H^{s}(\mathbb{R}), s>\frac{5}{2}$ has been established by employing a semigroup approach due to Kato [31] in [34], which, to our knowledge, is the only result on the Cauchy problem for the $\mathrm{fCH}$ equation.

In this paper, we refine the corresponding result in [34] by investigating the local wellposedness of the Cauchy problem for (1.1) in Besov space $B_{2,1}^{s_{0}}$ with $s_{0}=2 \nu-\frac{1}{2}$ for $\nu>\frac{3}{2}$ and $s_{0}=\frac{5}{2}$ for $1<\nu \leq \frac{3}{2}$. By virtue of the Littlewood-Paley decomposition, nonhomogeneous Besov spaces and iterative method, the methods proposed in [15, 16, 17] have been applied with success when studying the well-posedness of various shallow water wave equations in Besov space (see for example [20, 21, 23, 28, 32, 35, 36, 39]). To obtain our result, we felicitously recast the equation (1.1) in a form of nonlocal conservation law

$$
u_{t}+\frac{3}{5}(1+u) u_{x}=-\left(1+\frac{5}{4}\left(-\partial_{x}^{2}\right)^{\nu}\right)^{-1}\left(\frac{2}{5} u_{x}+\frac{2}{5} u u_{x}+\frac{1}{4}\left[u,\left(-\partial_{x}^{2}\right)^{\nu}\right] u_{x}\right)
$$

where [,] denotes the usual commutator of the linear operators. Then another difficulty arises as the appearance of commutator estimates of $\left[u,\left(-\partial_{x}^{2}\right)^{\nu}\right] u_{x}$ in $B_{2,1}^{s_{0}}$ and $B_{2, \infty}^{s_{0}-1}$. By the Bony decomposition, we break up the commutator term into the paraproduct terms and the remainder terms, which is beneficial to employing their continuity properties, and hence the desired estimates are obtained as presented in Lemma 3.4. Then we can establish a uniform bound for the approximate solutions on a sufficiently small timeinterval by employing the mean value theorem for integrals. This opens the path to obtain convergence and thus existence and uniqueness and continuous dependence on the initial datum are treated in separate steps of the proof.

Furthermore, provided that the initial profile $u_{0}$ is an analytic function on the real line $\mathbb{R}$, we obtain the analyticity of the corresponding solutions in both variables, with $x \in \mathbb{R}$ and $t$ in an interval around zero. Analyticity is inherent to travelling water waves (see [10]).

We supplement (1.1) with the initial data

$$
u(x, 0)=u_{0}(x), \quad x \in \mathbb{R} .
$$

To introduce the main results, we define

$$
E_{2,1}^{s_{0}}(T)=C\left([0, T] ; B_{2,1}^{s_{0}}\right) \cap C^{1}\left([0, T] ; B_{2,1}^{s_{0}-1}\right) .
$$

The main results of this paper are as follows: 
Theorem 1.1. Let $u_{0} \in B_{2,1}^{s_{0}}$ with $s_{0}=2 \nu-\frac{1}{2}$ for $\nu>\frac{3}{2}$ and $s_{0}=\frac{5}{2}$ for $1<\nu \leq \frac{3}{2}$. There exists a time $T>0$ such that the problem (1.1) and (1.4) has a unique solution in $E_{2,1}^{s_{0}}(T)$. Moreover, the solution depends continuously on the initial data, i.e., the mapping $\Phi: u_{0} \mapsto u$ is continuous from a neighborhood of $u_{0} \in B_{2,1}^{s_{0}}$ into $E_{2,1}^{s_{0}}(T)$.

Remark 1.1. We obtain the local well-posedness of equations (1.1) and (1.4) in the case $B_{2,1}^{s_{0}}$. However, this is not true in the case $B_{2, \infty}^{s_{0}}$ in view of the proof of Proposition 4 in [16]. Noting that $B_{2,1}^{s_{0}} \hookrightarrow H^{s_{0}} \hookrightarrow B_{2, \infty}^{s_{0}}$, one can see that $s=s_{0}$ is the critical index.

Referring to the definition of the space $E_{s}$ in (5.1), we present the following analytic result.

Theorem 1.2. If the initial data $u_{0}$ is real analytic on the line $\mathbb{R}$ and belongs to a space $E_{s}$ for some $0<s \leq 1$, then there exist an $\varepsilon>0$ and a unique solution $u$ to the problem (1.1) and (1.4) that is analytic on $\mathbb{R} \times[0, \varepsilon)$.

Remark 1.2. A rereading of the proof of Theorem 1.1 and Theorem 1.2 yields that there exists a real analytic extension of $u$ to $(-\varepsilon, \varepsilon)$.

In the sequel, we will, for notational convenience, deal with the following initial value problem with different coefficients, which implies the Theorem 1.1-Theorem 1.2 as the concrete values of the coefficients have no impact on the results.

$$
\left\{\begin{array}{l}
u_{t}+(1+u) u_{x}=\partial_{x} P(D) f_{1}(u)+P(D) f_{2}\left(u, u_{x}\right), \\
u(0, x)=u_{0}(x)
\end{array}\right.
$$

with the operator $P(D)=-\left(1+\left(-\partial_{x}^{2}\right)^{\nu}\right)^{-1}$, and

$$
f_{1}(u)=u+u^{2}, \quad f_{2}\left(u, u_{x}\right)=\left[u,\left(-\partial_{x}^{2}\right)^{\nu}\right] u_{x} .
$$

The rest of this paper is organized as follows. In Section 2, we give some preliminaries. Section 3 concentrates on the primary commutator estimates needed in the proof of Theorem 1.1 and Theorem 1.2. Section 4 aims at proving the local well-posedness for the Cauchy problem (1.1) and (1.4) in Besov space $B_{2,1}^{s_{0}}$. Section 5 is devoted to studying the analyticity of the Cauchy problem (1.1) and (1.4) based on a contraction type argument in a suitably chosen scale of the Banach spaces.

\section{PRELIMINARIES}

For convenience of the reader, we recall some conclusions on the properties of LittlewoodPaley decomposition, the nonhomogeneous Besov spaces and the theory of the transport equation. One may check [2, 15, 16, 17, 33] for more details. 
Lemma 2.1. (Littlewood-Paley decomposition). There exist two smooth radial functions $(\chi, \phi)$ valued in $[0,1]$, such that $\chi$ is supported in the ball $B=\left\{\xi \in \mathbb{R}^{n},|\xi| \leq \frac{4}{3}\right\}$ and $\phi$ is supported in the ring $C=\left\{\xi \in \mathbb{R}^{n}, \frac{4}{3} \leq|\xi| \leq \frac{8}{3}\right\}$. Moreover,

$$
\forall \xi \in \mathbb{R}^{n}, \quad \chi(\xi)+\sum_{q \geq 0} \phi\left(2^{-q} \xi\right)=1
$$

and

$$
\begin{gathered}
\operatorname{Supp} \phi\left(2^{-q} \cdot\right) \cap \operatorname{Supp} \phi\left(2^{-q^{\prime}} \cdot\right)=\emptyset, \quad \text { if }\left|q-q^{\prime}\right| \geq 2, \\
\operatorname{Supp} \chi(\cdot) \cap \operatorname{Supp} \phi\left(2^{-q} \cdot\right)=\emptyset, \quad \text { if }|q| \geq 1 .
\end{gathered}
$$

Then for $u \in \mathcal{S}^{\prime}\left(\mathbb{R}^{n}\right)$, the nonhomogeneous dyadic operators are defined as follows:

$$
\begin{aligned}
\triangle_{q} u & =0, \quad \text { if } q \leq-2, \\
\triangle_{-1} u & =\chi(\mathcal{D}) u=\mathscr{F}_{x}^{-1} \chi \mathscr{F}_{x} u, \\
\triangle_{q} u & =\phi\left(2^{-q} \mathcal{D}\right) u=\mathscr{F}_{x}^{-1} \phi\left(2^{-q} \xi\right) \mathscr{F}_{x} u, \quad \text { if } q \geq 0 .
\end{aligned}
$$

Thus $u=\sum_{q \geq 0} \triangle_{q} u$ in $\mathcal{S}^{\prime}\left(\mathbb{R}^{n}\right)$.

Remark 2.1. The low frequency cut-off $S_{q}$ is defined by

$$
S_{q} u=\sum_{p=-1}^{q-1} \triangle_{p} u=\chi\left(2^{-q} \mathcal{D}\right) u=\mathscr{F}_{x}^{-1} \chi\left(2^{-q} \xi\right) \mathscr{F}_{x} u, \quad \forall q \in N .
$$

It is easily checked that

$$
\begin{aligned}
\triangle_{p} \triangle_{q} & \equiv 0, \quad|p-q| \geq 2, \\
\triangle_{q}\left(S_{p-1} u \triangle_{p} v\right) & \equiv 0, \quad|p-q| \geq 5, \quad \forall u, v \in \mathcal{S}^{\prime}\left(\mathbb{R}^{n}\right)
\end{aligned}
$$

as well as

$$
\left\|\triangle_{q} u\right\|_{L^{p}} \leq\|u\|_{L^{p}}, \quad\left\|S_{q} u\right\|_{L^{p}} \leq C\|u\|_{L^{p}}, \quad \forall 1 \leq p \leq+\infty
$$

with the aid of Young's inequality, where $C$ is a positive constant independent of $q$.

Definition 2.1. (Besov spaces). Let $s \in \mathbb{R}, 1 \leq p \leq+\infty$. The nonhomogeneous Besov space $B_{p, r}^{s}\left(\mathbb{R}^{n}\right)$ is defined by

$$
B_{p, r}^{s}\left(\mathbb{R}^{n}\right)=\left\{f \in \mathcal{S}^{\prime}\left(\mathbb{R}^{n}\right):\|f\|_{B_{p, r}^{s}}=\left\|2^{q s} \triangle_{q} f\right\|_{l^{r}\left(L^{P}\right)}=\left\|\left(2^{q s}\left\|\triangle_{q} f\right\|_{L^{p}}\right)_{q \geq-1}\right\|_{l^{r}}<\infty\right\} .
$$

In particular, $B_{p, r}^{\infty}=\bigcap_{s \in \mathbb{R}} B_{p, r}^{s}$.

Lemma 2.2. Let $s \in \mathbb{R}, 1 \leq p, r, p_{j}, r_{j} \leq \infty, j=1,2$, then:

(1) Topological properties: $B_{p, r}^{s}$ is a Banach space which is continuously embedded in $\mathcal{S}^{\prime}$.

(2) Density: $C_{c}^{\infty}$ is dense in $B_{p, r}^{s} \Leftrightarrow 1 \leq p, r<\infty$.

(3) Embedding: $B_{p, r}^{s} \hookrightarrow B_{p, \tilde{r}}^{\tilde{r}}$, if $\tilde{s}<s$ or $\tilde{s}=s$ and $\tilde{r} \geq r . B_{p_{1}, r_{1}}^{s} \hookrightarrow B_{p_{2}, r_{2}}^{s-n\left(\frac{1}{p_{1}}-\frac{1}{p_{2}}\right)}$, if $p_{1} \leq p_{2}$ and $r_{1} \leq r_{2}$ and $B_{p, 1}^{0} \hookrightarrow L^{p} \hookrightarrow B_{p, \infty}^{0}$. 
(4) Algebraic properties: $\forall s>0, B_{p, r}^{s} \cap L^{\infty}$ is a Banach algebra. $B_{p, r}^{s}$ is a Banach algebra $\Leftrightarrow B_{p, r}^{s} \hookrightarrow L^{\infty} \Leftrightarrow s>\frac{1}{p}$ or $\left(s \geq \frac{1}{p}\right.$ and $\left.r=1\right)$. In particular, $B_{2,1}^{1 / 2}$ is continuously embedded in $B_{2, \infty}^{1 / 2} \cap L^{\infty}$ and $B_{2, \infty}^{1 / 2} \cap L^{\infty}$ is a Banach algebra.

(5) 1-D Moser-type estimates:

(i) For $s>0$,

$$
\|f g\|_{B_{p, r}^{s}} \leq C\left(\|f\|_{B_{p, r}^{s}}\|g\|_{L^{\infty}}+\|g\|_{B_{p, r}^{s}}\|f\|_{L^{\infty}}\right) .
$$

(ii) $\forall s_{1} \leq \frac{1}{p}<s_{2}\left(s_{2} \geq \frac{1}{p}\right.$ if $\left.r=1\right)$ and $s_{1}+s_{2}>0$, we have

$$
\|f g\|_{B_{p, r}^{s_{1}}} \leq C\|f\|_{B_{p, r}^{s_{1}}}\|g\|_{B_{p, r}^{s_{2}}} .
$$

(6) Complex interpolation:

$$
\|f\|_{B_{p, r}^{\theta s_{1}+(1-\theta) s_{2}}} \leq\|f\|_{B_{p, r}^{s_{1}}}^{\theta}\|f\|_{B_{p, r}^{s_{2}},}^{1-\theta}, \quad \forall f \in B_{p, r}^{s_{1}} \cap B_{p, r}^{s_{2}}, \quad \forall \theta \in[0,1] .
$$

(7) Real interpolation: $\forall \theta \in(0,1), s_{1}<s_{2}, s=\theta s_{1}+(1-\theta) s_{2}$, there exists a constant C such that

$$
\|u\|_{B_{p, 1}^{s}} \leq \frac{C(\theta)}{s_{2}-s_{1}}\|u\|_{B_{p, \infty}^{s_{1}}}^{\theta}\|u\|_{B_{p, \infty}^{s_{2}}}^{1-\theta}, \forall u \in B_{p, \infty}^{s_{1}} .
$$

In particular, for any $0<\theta<1$, we have

$$
\|u\|_{B_{2,1}^{s_{0}-1}} \leq\|u\|_{B_{2,1}^{s_{0}-\theta}} \leq C(\theta)\|u\|_{B_{2, \infty}^{s_{0}-1}}^{\theta}\|u\|_{B_{2, \infty}^{s_{0}}}^{1-\theta} .
$$

(8) Fatou lemma: if $\left(u_{n}\right)_{n \in \mathbb{N}}$ is bounded in $B_{p, r}^{s}$ and $u_{n} \rightarrow u$ in $\mathcal{S}^{\prime}$, then $u \in B_{p, r}^{s}$ and

$$
\|u\|_{B_{p, r}^{s}} \leq \liminf _{n \rightarrow \infty}\left\|u_{n}\right\|_{B_{p, r}^{s}} .
$$

(9) Let $m \in \mathbb{R}$ and $f$ be an $s^{m}$-multiplier (i.e., $f: \mathbb{R}^{n} \rightarrow \mathbb{R}$ is smooth and satisfies that $\forall \alpha \in N^{n}, \exists$ a constant $C_{\alpha}$, s.t. $\left|\partial_{\alpha} f(\xi)\right| \leq C_{\alpha}(1+|\xi|)^{m-|\alpha|}$ for all $\left.\xi \in \mathbb{R}^{n}\right)$. Then the operator $f(D)$ is continuous from $B_{p, r}^{s}$ to $B_{p, r}^{s-m}$.

(10) The paraproduct is continuous from $B_{p, 1}^{-1 / p} \times\left(B_{p, \infty}^{1 / p} \cap L^{\infty}\right)$ to $B_{p, 1}^{-1 / p}$, i.e.,

$$
\|f g\|_{B_{p, \infty}^{-1 / p}} \leq C\|f\|_{B_{p, 1}^{-1 / p}}\|g\|_{B_{p, \infty}^{1 / p} \cap L^{\infty}} .
$$

(11) A logarithmic interpolation inequality

$$
\|f\|_{B_{p, 1}^{s}} \leq C\|f\|_{B_{p, \infty}^{s}} \ln \left(e+\frac{\|f\|_{B_{p, \infty}^{s+1}}}{\|f\|_{B_{p, \infty}^{s}}}\right) .
$$

Lemma 2.3. Let $1 \leq p, r \leq \infty$ and $s>-\min \left(\frac{1}{p}, 1-\frac{1}{p}\right)$. Assume that $f_{0} \in B_{p, r}^{s}$, $F \in L^{1}\left(0, T ; B_{p, r}^{s}\right)$ and $\partial_{x} v$ belongs to $L^{1}\left(0, T ; B_{p, r}^{s-1}\right)$ if $s>1+\frac{1}{p}$ or to $L^{1}\left(0, T ; B_{p, r}^{1 / p} \cap\right.$ $\left.L^{\infty}\right)$ otherwise. If $f \in L^{\infty}\left(0, T ; B_{p, r}^{s}\right) \cap C\left([0, T] ; \mathcal{S}^{\prime}(\mathbb{R})\right)$ solves the following 1-D linear transport equation:

$$
\left\{\begin{array}{l}
f_{t}+v f_{x}=F \\
\left.f\right|_{t=0}=f_{0} .
\end{array}\right.
$$

then there exists a constant $C$ depending only on $s, p, r$ such that the following statements hold: 
(1) If $r=1$ or $s \neq 1+\frac{1}{p}$, then

$$
\|f\|_{B_{p, r}^{s}} \leq\left\|f_{0}\right\|_{B_{p, r}^{s}}+\int_{0}^{t}\|F(\tau)\|_{B_{p, r}^{s}} d \tau+C \int_{0}^{t} V^{\prime}(\tau)\|f(\tau)\|_{B_{p, r}^{s}} d \tau,
$$

or

$$
\|f\|_{B_{p, r}^{s}} \leq e^{C V(t)}\left(\left\|f_{0}\right\|_{B_{p, r}^{s}}+\int_{0}^{t} e^{-C V(\tau)}\|F(\tau)\|_{B_{p, r}^{s}} d \tau\right)
$$

holds, where $V(t)=\int_{0}^{t}\left\|v_{x}(\tau)\right\|_{B_{p, r}^{1 / p} \cap L^{\infty}} d \tau$ if $s<1+\frac{1}{p}$ and $V(t)=\int_{0}^{t}\left\|v_{x}(\tau)\right\|_{B_{p, r}^{s-1}} d \tau$ else.

(2) If $s \leq 1+\frac{1}{p}, f_{0}^{\prime} \in L^{\infty}, f_{x} \in L^{\infty}((0, t) \times \mathbb{R})$ and $F_{x} \in L^{1}\left(0, T ; L^{\infty}\right)$, then

$$
\begin{aligned}
& \|f(t)\|_{B_{p, r}^{s}}+\left\|f_{x}(t)\right\|_{L^{\infty}} \\
& \quad \leq e^{C V(t)}\left(\left\|f_{0}\right\|_{B_{p, r}^{s}}+\left\|f_{0 x}\right\|_{L^{\infty}}+\int_{0}^{t} e^{-C V(\tau)}\left[\|F(\tau)\|_{B_{p, r}^{s}}+\left\|F_{x}(\tau)\right\|_{L^{\infty}}\right] d \tau\right)
\end{aligned}
$$

with $V(t)=\int_{0}^{t}\left\|v_{x}(\tau)\right\|_{B_{p, r}^{1 / p} \cap L^{\infty}} d \tau$.

(3) If $f=v$, then for all $s>0$, the estimate (2.2) holds with $V(t)=\int_{0}^{t}\left\|v_{x}(\tau)\right\|_{L^{\infty}} d \tau$.

(4) If $r<\infty$, then $f \in C\left([0, T] ; B_{p, r}^{s}\right)$. If $r=\infty$, then $f \in C\left([0, T] ; B_{p, r}^{s^{\prime}}\right)$ for all $s^{\prime}<s$.

Lemma 2.4. (Existence and uniqueness) Let $p, r, s, f_{0}$ and $F$ be as in the statement of Lemma 2.3 Assume that $v \in L^{\rho}\left(0, t ; B_{\infty, \infty}^{-M}\right)$ for some $\rho>1$ and $M>0$ and $v_{x} \in$ $L^{1}\left(0, T ; B_{p, r}^{s-1}\right)$ if $s>1+\frac{1}{p}$ or $s=1+\frac{1}{p}$ and $r=1$ and $v_{x} \in L^{1}\left(0, T ; B_{p, \infty}^{1 / p} \cap L^{\infty}\right)$ if $<1+\frac{1}{p}$. Then the transport equation (T) has a unique solution $f \in L^{\infty}\left([0, T] ; B_{p, r}^{s}\right) \cap$ $\left(\bigcap_{s^{\prime}<s} C\left([0, T] ; B_{p, 1}^{s^{\prime}}\right)\right.$ and the inequalities in Lemma 2.3 hold true. Moreover, if $r<\infty$, then we have $f \in C\left([0, T] ; B_{p, r}^{s}\right)$.

Lemma 2.5. ([16]) Denote $\overline{\mathbb{N}}=\mathbb{N} \cup \infty$. Let $\left(v^{(n)}\right)_{n \in \overline{\mathbb{N}}}$ be a sequence of functions belonging to $C\left([0, T] ; B_{2,1}^{1 / 2}\right)$. Assume that $v^{(n)}$ is the solution to

$$
\left\{\begin{array}{l}
\partial_{t} v^{(n)}+a^{(n)} \partial_{x} v^{(n)}=f \\
\left.v^{(n)}\right|_{t=0}=v_{0}
\end{array}\right.
$$

with $v_{0} \in B_{2,1}^{1 / 2}, f \in L^{1}\left(0, T ; B_{2,1}^{1 / 2}\right)$ and that for some $\alpha \in L^{1}(0, T)$,

$$
\sup _{n \in \mathbb{N}}\left\|\partial_{x} a^{(n)}(t)\right\|_{B_{2,1}^{1 / 2}} \leq \alpha(t)
$$

If in addition $a^{(n)}$ tends to $a^{(\infty)}$ in $L^{1}\left(0, T ; B_{2,1}^{1 / 2}\right)$ then $v^{(n)}$ tends to $v^{(\infty)}$ in $C\left(0, T ; B_{2,1}^{1 / 2}\right)$.

\section{Commutator estimates}

This section is devoted to the vital commutator estimates needed in the proof of Theorem 1.1 and Theorem 1.2. To begin with, we give the definition of the Bony decomposition. 
Definition 3.1. [2] The nonhomogeneous paraproduct of $v$ by $u$ is defined by

$$
T_{u} v \stackrel{\text { def }}{=} \sum_{j} S_{j-1} u \Delta_{j} v
$$

and the nonhomogeneous remainder of $u$ and $v$ is defined by

$$
R(u, v)=\sum_{|k-j| \leq 1} \Delta_{k} u \Delta_{j} v
$$

Then the Bony decomposition is given by

$$
u v=T_{u} v+T_{v} u+R(u, v) .
$$

or

$$
u v=T_{u} v+T_{v}^{\prime} u \quad \text { with } \quad T_{v}^{\prime} u \stackrel{\text { def }}{=} \sum_{j} S_{j+2} v \Delta_{j} u
$$

The required properties which will be used in the proof the commutator estimates are given by the following lemmas.

Lemma 3.1. [2] Let $f$ be a smooth function on $\mathbb{R}$. Assume that $f$ is homogeneous of degree $m$ away from a neighborhood of 0 . Let $\rho$ be in $(0,1)$, $s$ be in $\mathbb{R}$, and $(p, r)$ be in $[1, \infty]^{2}$. There exists a constant $C$, depending only on $s$ and $\rho$, such that if $\left(p_{1}, p_{2}\right) \in$ $[1, \infty]^{2}$ satisfies $\frac{1}{p}=\frac{1}{p_{1}}+\frac{1}{p_{2}}$, then the following estimate holds true:

$$
\left\|\left[T_{a}, f(D)\right] u\right\|_{B_{p, r}^{s-m+\rho}} \leq C\left\|\partial_{x} a\right\|_{B_{p_{1}, \infty}^{\rho-1}}\|u\|_{B_{p_{2}, r}^{s}} .
$$

In the limit case $\rho=1$, we have

$$
\left\|\left[T_{a}, f(D)\right] u\right\|_{B_{p, r}^{s-m+1}} \leq C\left\|\partial_{x} a\right\|_{L^{p_{1}}}\|u\|_{B_{p_{2}, r}^{s}} .
$$

Lemma 3.2. [2] There exists a constant $C>0$, such that for any $s \in \mathbb{R}, \sigma>0$ and $1 \leq p, r_{1}, r_{2} \leq \infty$, it holds that

$$
\left\|T_{u} v\right\|_{B_{p, r}^{s}} \leq C^{|s|+1}\|u\|_{L^{\infty}}\|v\|_{B_{p, r}^{s}}
$$

and

$$
\left\|T_{u} v\right\|_{B_{p, r_{1,2}}^{s-\sigma}} \leq \frac{C^{|s-\sigma|+1}}{\sigma}\|u\|_{B_{\infty, r_{1}}^{-\sigma}}\|v\|_{B_{p, r_{2}}^{s}}, \quad \text { with } \frac{1}{r_{1,2}}=\min \left(1, \frac{1}{r_{1}}+\frac{1}{r_{2}}\right) \text {. }
$$

Lemma 3.3. [17, 33] Let $s_{1}, s_{2} \in \mathbb{R}$ and $1 \leq p, p_{1}, p_{2}, r_{1}, r_{2} \leq \infty$. Assume that

$$
\frac{1}{p} \leq \frac{1}{p_{1}}+\frac{1}{p_{2}} \leq 1, \quad \sigma_{1,2}-\frac{d}{p}=\left(s_{1}-\frac{d}{p_{1}}\right)+\left(s_{2}-\frac{d}{p_{2}}\right) .
$$

Then there exists a constant $C>0$ such that

(1) If

$$
s_{1}+s_{2}>0, \quad \frac{1}{r_{1}}+\frac{1}{r_{2}} \stackrel{\text { def }}{=} \frac{1}{r} \leq 1
$$


then we have

$$
\|R(u, v)\|_{B_{p, r}^{\sigma \sigma_{1}}} \leq \frac{C^{\left|s_{1}+s_{2}\right|+1}}{s_{1}+s_{2}}\|u\|_{B_{p_{1}, r_{1}}^{s_{1}}}\|v\|_{B_{p_{2}, r_{2}}^{s_{2}}}
$$

(2) If

$$
s_{1}+s_{2}=0, \quad \frac{1}{r_{1}}+\frac{1}{r_{2}}=1
$$

then we have

$$
\|R(u, v)\|_{B_{p, \infty}^{\sigma_{1,2}}} \leq C^{\left|s_{1}+s_{2}\right|+1}\|u\|_{B_{p_{1}, r_{1}}^{s_{1}}}\|v\|_{B_{p_{2}, r_{2}}^{s_{2}}} .
$$

Now we are in the position to give the desired commutator estimates.

Lemma 3.4. There exists a constant $C>0$ such that

$$
\begin{gathered}
\left\|\left[f,\left(-\partial_{x}^{2}\right)^{\nu}\right] g\right\|_{B_{2,1}^{s_{0}-2 \nu}} \leq C\|f\|_{B_{2,1}^{s_{0}}}\|g\|_{B_{2,1}^{s_{0}-1}} \quad \text { for } f \in B_{2,1}^{s_{0}}, g \in B_{2,1}^{s_{0}-1}, \\
\left\|\left[f,\left(-\partial_{x}^{2}\right)^{\nu}\right] g\right\|_{B_{2, \infty}^{s_{0}-1-2 \nu}} \leq C\|f\|_{B_{2,1}^{s_{0}-1}}\|g\|_{B_{2,1}^{s_{0}-1}} \quad \text { for } f \in B_{2,1}^{s_{0}-1}, g \in B_{2,1}^{s_{0}-1},
\end{gathered}
$$

and

$$
\left\|\left[f,\left(-\partial_{x}^{2}\right)^{\nu}\right] g\right\|_{B_{2, \infty}^{s_{0}-1-2 \nu}} \leq C\|f\|_{B_{2,1}^{s_{0}}}\|g\|_{B_{2,1}^{s_{0}-2}} \quad \text { for } f \in B_{2,1}^{s_{0}}, g \in B_{2,1}^{s_{0}-2}
$$

where $s_{0}=2 \nu-\frac{1}{2}$ for $\nu>\frac{3}{2}$ and $s_{0}=\frac{5}{2}$ for $1<\nu \leq \frac{3}{2}$.

Proof. We write $\left[f,\left(-\partial_{x}^{2}\right)^{\nu}\right] g=F+G$ with

$$
F \stackrel{\text { def }}{=}\left[T_{f},\left(-\partial_{x}^{2}\right)^{\nu}\right] g+T_{\left(-\partial_{x}^{2}\right)^{\nu} g} f-\left(-\partial_{x}^{2}\right)^{\nu} T_{g}^{\prime} f
$$

and

$$
G \stackrel{\text { def }}{=} R\left(f,\left(-\partial_{x}^{2}\right)^{\nu} g\right)
$$

First, we give the proof of (3.14). Taking $s=s_{0}-1, m=2 \nu, p=2, r=1, p_{1}=\infty, p_{2}=$ 2 in (3.6), we have

$$
\begin{aligned}
\left\|\left[T_{f},\left(-\partial_{x}^{2}\right)^{\nu}\right] g\right\|_{B_{2,1}^{s_{0}-2 \nu}} & =\left\|\left[T_{f},\left(-\partial_{x}^{2}\right)^{\nu}\right] g\right\|_{B_{2,1}^{s_{0}-1-2 \nu+1}} \\
& \leq C\left\|f_{x}\right\|_{L^{\infty}}\|g\|_{B_{2,1}^{s_{0}-1}} \leq C\|f\|_{B_{2,1}^{s_{0}}}\|g\|_{B_{2,1}^{s_{0}-1}},
\end{aligned}
$$

where the algebraic properties (4) in Lemma 2.2 have been used. Taking $s=s_{0}, \sigma=$ $2 \nu, p=2, r_{1}=1, r_{2}=\infty$ in (3.8), we obtain

$$
\begin{aligned}
\left\|T_{\left(-\partial_{x}^{2}\right)^{\nu} g} f\right\|_{B_{2,1}^{s_{0}-2 \nu}} & \leq C\left\|\left(-\partial_{x}^{2}\right)^{\nu} g\right\|_{B_{\infty, \infty}^{-2 \nu}}\|f\|_{B_{2,1}^{s_{0}}} \\
& \leq C\|g\|_{L^{\infty}}\|f\|_{B_{2,1}^{s_{0}}} \leq C\|f\|_{B_{2,1}^{s_{0}}}\|g\|_{B_{2,1}^{s_{0}-1}},
\end{aligned}
$$

where the property $L^{\infty} \hookrightarrow B_{\infty, \infty}^{0}$ has been used. By (3.7) and (3.11), we get

$$
\begin{aligned}
\left\|\left(-\partial_{x}^{2}\right)^{\nu} T_{g}^{\prime} f\right\|_{B_{2,1}^{s_{0}-2 \nu}} \leq C\left\|T_{g}^{\prime} f\right\|_{B_{2,1}^{s_{0}}} & \leq C\left\|T_{g} f\right\|_{B_{2,1}^{s_{0}}}+C\|R(f, g)\|_{B_{2,1}^{s_{0}}} \\
& \leq C\|g\|_{L^{\infty}}\|f\|_{B_{2,1}^{s_{0}}} \leq C\|f\|_{B_{2,1}^{s_{0}}}\|g\|_{B_{2,1}^{s_{0}-1}} .
\end{aligned}
$$


Taking $s_{1}=s_{0}, s_{2}=-\frac{3}{2}, p=2, p_{1}=2, p_{2}=2, r=2, r_{1}=2, r_{2}=2, \sigma_{1,2}=s_{0}-2$ in (3.11, we obtain

$$
\begin{aligned}
\left\|R\left(f,\left(-\partial_{x}^{2}\right)^{\nu} g\right)\right\|_{B_{2,1}^{s_{0}-2 \nu}} & \leq C\left\|R\left(f,\left(-\partial_{x}^{2}\right)^{\nu} g\right)\right\|_{B_{2,1}^{s_{0}-2}} \\
& \leq C\|f\|_{B_{2,2}^{s_{0}}}\left\|\left(-\partial_{x}^{2}\right)^{\nu} g\right\|_{B_{2,2}^{-\frac{3}{2}}} \leq C\|f\|_{B_{2,1}^{s_{0}}}\|g\|_{B_{2,1}^{s_{0}-1}} .
\end{aligned}
$$

Combing (3.17)-(3.22) leads to (3.14). Now, we turn to the proof of (3.15) and (3.16). Taking $s=s_{0}-2, m=2 \nu, p=2, r=\infty, p_{1}=\infty, p_{2}=2$ in (3.6), we have

$$
\begin{aligned}
\left\|\left[T_{f},\left(-\partial_{x}^{2}\right)^{\nu}\right] g\right\|_{B_{2, \infty}^{s_{0}-1-2 \nu}} & =\left\|\left[T_{f},\left(-\partial_{x}^{2}\right)^{\nu}\right] g\right\|_{B_{2, \infty}^{s_{0}-2-2 \nu+1}} \\
& \leq C\left\|f_{x}\right\|_{L^{\infty}}\|g\|_{B_{2, \infty}^{s_{0}-2}} \leq C\|f\|_{B_{2,1}^{s_{0}-1}}\|g\|_{B_{2,1}^{s_{0}-2}} .
\end{aligned}
$$

Taking $s=s_{0}-1, \sigma=2 \nu, p=2, r_{1}=\infty, r_{2}=\infty$ in (3.8), we obtain

$$
\begin{aligned}
\left\|T_{\left(-\partial_{x}^{2}\right)^{\nu} g} f\right\|_{B_{2, \infty}^{s_{0}-1-2 \nu}} & \leq C\left\|\left(-\partial_{x}^{2}\right)^{\nu} g\right\|_{B_{\infty}^{-2 \nu},}\|f\|_{B_{2, \infty}^{s_{0}-1}} \\
& \leq C\|g\|_{L^{\infty}}\|f\|_{B_{2, \infty}^{s_{0}-1}} \leq C\|f\|_{B_{2,1}^{s_{0}-1}}\|g\|_{B_{2,1}^{s_{0}-2} .}
\end{aligned}
$$

By (3.7) and 3.11), we get

$$
\begin{aligned}
\left\|\left(-\partial_{x}^{2}\right)^{\nu} T_{g}^{\prime} f\right\|_{B_{2, \infty}^{s_{0}-1-2 \nu}} & \leq C\left\|T_{g}^{\prime} f\right\|_{B_{2, \infty}^{s_{0}-1}} \\
& \leq C\|g\|_{L^{\infty}}\|f\|_{B_{2, \infty}^{s_{0}-1}} \leq C\|f\|_{B_{2,1}^{s_{0}-1}}\|g\|_{B_{2,1}^{s_{0}-2}}
\end{aligned}
$$

This completes the estimate of the term $F$, and we now give the estimate of the term $G$.

(1) Assume $\nu>\frac{3}{2}$ and thus $s_{0}=2 \nu-\frac{1}{2}$.

(i) Taking $s_{1}=s_{0}-1, s_{2}=-\frac{3}{2}, p=2, p_{1}=2, p_{2}=2, r=\infty, r_{1}=\infty, r_{2}=$ $\infty, \sigma_{1,2}=s_{0}-3$ in (3.11), we obtain

$$
\begin{aligned}
\left\|R\left(f,\left(-\partial_{x}^{2}\right)^{\nu} g\right)\right\|_{B_{2, \infty}^{s_{0}-1-2 \nu}} & \leq C\left\|R\left(f,\left(-\partial_{x}^{2}\right)^{\nu} g\right)\right\|_{B_{2, \infty}^{s_{0}-3}} \\
& \leq C\|f\|_{B_{2, \infty}^{s_{0}-1}}\left\|\left(-\partial_{x}^{2}\right)^{\nu} g\right\|_{B_{2, \infty}^{-\frac{3}{2}}} \leq C\|f\|_{B_{2,1}^{s_{0}-1}}\|g\|_{B_{2,1}^{s_{0}-1}} .
\end{aligned}
$$

(ii) Taking $s_{1}=s_{0}, s_{2}=-\frac{5}{2}, p=2, p_{1}=2, p_{2}=2, r_{1}=\infty, r_{2}=\infty, \sigma_{1,2}=s_{0}-3$ in (3.11), we obtain

$$
\begin{aligned}
\left\|R\left(f,\left(-\partial_{x}^{2}\right)^{\nu} g\right)\right\|_{B_{2, \infty}^{s_{0}-1-2 \nu}} & \leq C\left\|R\left(f,\left(-\partial_{x}^{2}\right)^{\nu} g\right)\right\|_{B_{2, \infty}^{s_{0}-3}} \\
& \leq C\|f\|_{B_{2, \infty}^{s_{0}}}\left\|\left(-\partial_{x}^{2}\right)^{\nu} g\right\|_{B_{2, \infty}^{-\frac{5}{2}}} \leq C\|f\|_{B_{2,1}^{s_{0}}}\|g\|_{B_{2,1}^{s_{0}-2} .}
\end{aligned}
$$

(2) Assume $1<\nu \leq \frac{3}{2}$ and thus $s_{0}=\frac{5}{2}$.

(i) Taking $s_{1}=\frac{3}{2}, s_{2}=-\frac{3}{2}, p=2, p_{1}=2, p_{2}=2, r_{1}=1, r_{2}=\infty, \sigma_{1,2}=-\frac{1}{2}$ in (3.12), we obtain

$$
\begin{aligned}
\left\|R\left(f,\left(-\partial_{x}^{2}\right)^{\nu} g\right)\right\|_{B_{2, \infty}^{\frac{3}{2}-2 \nu}} & \leq C\left\|R\left(f,\left(-\partial_{x}^{2}\right)^{\nu} g\right)\right\|_{B_{2, \infty}^{-\frac{1}{2}}} \\
& \leq C\|f\|_{B_{2,1}^{\frac{3}{2}}}\left\|\left(-\partial_{x}^{2}\right)^{\nu} g\right\|_{B_{2, \infty}^{-\frac{3}{2}}} \leq C\|f\|_{B_{2,1}^{s_{0}-1}}\|g\|_{B_{2,1}^{s_{0}-1}}
\end{aligned}
$$


(ii) Taking $s_{1}=\frac{5}{2}, s_{2}=-\frac{5}{2}, p=2, p_{1}=2, p_{2}=2, r_{1}=1, r_{2}=\infty, \sigma_{1,2}=-\frac{1}{2}$ in (3.12), we obtain

$$
\begin{aligned}
\left\|R\left(f,\left(-\partial_{x}^{2}\right)^{\nu} g\right)\right\|_{B_{2, \infty}^{\frac{3}{2}-2 \nu}} & \leq C\left\|R\left(f,\left(-\partial_{x}^{2}\right)^{\nu} g\right)\right\|_{B_{2, \infty}^{-\frac{1}{2}}} \\
& \leq C\|f\|_{B_{2,1}^{\frac{5}{2}}}\left\|\left(-\partial_{x}^{2}\right)^{\nu} g\right\|_{B_{2, \infty}^{-\frac{5}{2}}} \leq C\|f\|_{B_{2,1}^{s_{0}}}\|g\|_{B_{2,1}^{s_{0}-2} .}
\end{aligned}
$$

Combing (3.23)-(3.25) with (3.17), (3.18), (3.26), (3.28) yields (3.15) and combing (3.23)(3.25) with (3.17), (3.18), (3.27), (3.29) yields (3.16). This completes the proof of Lemma 3.4 .

\section{Proof of Theorem 1.1}

In this section we aim to prove Theorem 1.1 with the aid of the following six steps.

First step: Approximate solution. We use a standard iterative process to build a solution. Starting from $u^{(0)}:=0$, by induction we define a sequence of smooth functions $\left(u^{(n)}\right)_{n \in \mathbb{N}}$ by solving the following linear transport equation:

$$
\left\{\begin{array}{l}
u_{t}^{(n+1)}+\left(1+u^{(n)}\right) u_{x}^{(n+1)}=\partial_{x} P(D) f_{1}\left(u^{(n)}\right)+P(D) f_{2}\left(u^{(n)}, u_{x}^{(n)}\right), \\
u^{(n+1)}(0, x)=u_{0}^{(n+1)}(x)=S_{n+1} u_{0} .
\end{array}\right.
$$

Since $S_{n+1} u_{0}$ belongs to $B_{2, r}^{\infty}$, by using Lemma 2.4, with the aid of induction, we show that for all $n \in \mathbb{N}$, the above equation has a global solution which belongs to $C\left(\mathbb{R}^{+}, B_{2, r}^{\infty}\right)$.

Second step: Uniform bounds. Applying (2.2) of Lemma 2.3 to (4.1), we obtain

$$
\begin{aligned}
& \left\|u^{(n+1)}(t)\right\|_{B_{2,1}^{s_{0}}} \leq e^{C \int_{0}^{t}\left\|u^{(n)}\right\|_{B_{2,1}^{s_{0}}} d t^{\prime}}\left\|u_{0}\right\|_{B_{2,1}^{s_{0}}} \\
& +\int_{0}^{t} e^{C \int_{\tau}^{t}\left\|u^{(n)}\right\|_{B_{2,1}^{s_{0}} d t^{\prime}}}\left\|\partial_{x} P(D) f_{1}\left(u^{(n)}\right)+P(D) f_{2}\left(u^{(n)}, u_{x}^{(n)}\right)\right\|_{B_{2,1}^{s_{0}}} d \tau .
\end{aligned}
$$

As $P(D)$ is a $S^{-2 \nu}$-multiplier and $B_{2, r}^{s} \hookrightarrow B_{2, r}^{s-1}$, we have that

$$
\begin{aligned}
\left\|\partial_{x} P(D) f_{1}\left(u^{(n)}\right)\right\|_{B_{2,1}^{s_{0}}} & \leq\left\|f_{1}\left(u^{(n)}\right)\right\|_{B_{2,1}^{s_{0}-2 \nu+1}} \leq\left\|f_{1}\left(u^{(n)}\right)\right\|_{B_{2,1}^{s_{0}-1}} \\
& \leq \frac{C}{2}\left(\left\|u^{(n)}\right\|_{B_{2,1}^{s_{0}}}+\left\|u^{(n)}\right\|_{B_{2,1}^{s_{0}}}^{2}\right)
\end{aligned}
$$

and by (3.14) of lemma 3.4 and $B_{2,1}^{1 / 2} \hookrightarrow L^{\infty}$, we have

$$
\left\|P(D) f_{2}\left(u^{(n)}, u_{x}^{(n)}\right)\right\|_{B_{2,1}^{s_{0}}} \leq C\left\|\left[u^{(n)},\left(-\partial_{x}^{2}\right)^{\nu}\right] u_{x}^{(n)}\right\|_{B_{2,1}^{s_{0}-2 \nu}} \leq \frac{C}{2}\left\|u^{(n)}\right\|_{B_{2,1}^{s_{0}}}^{2} .
$$

Inserting (4.3) and (4.4) into (4.2) yields for all $n \in \mathbb{N}$ :

$$
\begin{aligned}
\left\|u^{(n+1)}(t)\right\|_{B_{2,1}^{s_{0}}} & \leq e^{C \int_{0}^{t}\left\|u^{(n)}\left(t^{\prime}\right)\right\|_{B_{2,1}^{s_{0}}} d t^{\prime}}\left\|u_{0}\right\|_{B_{2,1}^{s_{0}}} \\
& +\frac{C}{2} \int_{0}^{t} e^{C \int_{\tau}^{t}\left\|u^{(n)}\left(t^{\prime}\right)\right\|_{B_{2,1}^{s_{0}}} d t^{\prime}}\left(\left\|u^{(n)}\right\|_{B_{2,1}^{s_{0}}}+\left\|u^{(n)}\right\|_{B_{2,1}^{s_{0}}}^{2}\right) d \tau .
\end{aligned}
$$


Let us choose a $T>0$ such that

$$
T \leq \min \left\{\frac{1}{C}, \frac{1}{8 C\left\|u_{0}\right\|_{B_{2,1}^{s_{0}}}}\right\},
$$

and suppose by induction that for all $t \in[0, T]$

$$
\left\|u^{(n)}(t)\right\|_{B_{2,1}^{s_{0}}} \leq \frac{2\left\|u_{0}\right\|_{B_{2,1}^{s_{0}}}}{1-4 C\left\|u_{0}\right\|_{B_{2,1}^{s_{0}} t}} .
$$

Indeed, one obtains from (4.5) and (4.7) that

$$
\begin{aligned}
\exp \left\{C \int_{\tau}^{t}\left\|\left(u^{(n)}\right)\left(t^{\prime}\right)\right\|_{B_{2,1}^{s_{0}}} d t^{\prime}\right\} & \leq \exp \left\{\int_{\tau}^{t} \frac{2 C\left\|u_{0}\right\|_{B_{2,1}^{s_{0}}}}{1-4 C\left\|u_{0}\right\|_{B_{2,1}^{s_{0}} t^{\prime}}} d t^{\prime}\right\} \\
& =\exp \left\{-\frac{1}{2} \int_{\tau}^{t} \frac{d\left(1-4 C\left\|u_{0}\right\|_{B_{2,1}^{s_{0}}} t^{\prime}\right)}{\left(1-4 C\left\|u_{0}\right\|_{\left.B_{2,1}^{s_{0}} t^{\prime}\right)}\right.}\right\} \\
& =\exp \left\{\frac{1}{2} \ln \left(\frac{1-4 C\left\|u_{0}\right\|_{B_{2,1}^{s_{0}} \tau}}{1-4 C\left\|u_{0}\right\|_{B_{2,1}^{s_{0}} t}}\right)\right\} \\
& =\left(\frac{1-4 C\left\|u_{0}\right\|_{B_{2,1}^{s_{0}} \tau}}{1-4 C\left\|u_{0}\right\|_{B_{2,1}^{s_{0}} t}^{\frac{1}{2}}} .\right.
\end{aligned}
$$

When $\tau=0$, we have

$$
e^{C \int_{0}^{t}\left\|\left(u^{(n)}\right)\left(t^{\prime}\right)\right\|_{B_{2,1}^{s_{0}} d t^{\prime}}} \leq \frac{1}{\left(1-4 C\left\|u_{0}\right\|_{\left.B_{2,1}^{s_{0}} t\right)^{\frac{1}{2}}}\right.}
$$

Then combining (4.7) and (4.8), we have

$$
\begin{aligned}
& \frac{C}{2} \int_{0}^{t} e^{C \int_{\tau}^{t}\left\|\left(u^{(n)}\right)\left(t^{\prime}\right)\right\|_{B_{2,1}^{s_{0}}} d t^{\prime}}\left(\left\|u^{(n)}\right\|_{B_{2,1}^{s_{0}}}+\left\|u^{(n)}\right\|_{B_{2,1}^{s_{0}}}^{2}\right) d \tau \\
& \leq \frac{\left\|u_{0}\right\|_{B_{2,1}^{s_{0}}}}{\left(1-4 C\left\|u_{0}\right\|_{\left.B_{2,1}^{s_{0}} t\right)^{1 / 2}}\right.} \\
& \times \int_{0}^{t}\left(1-4 C\left\|u_{0}\right\|_{B_{2,1}^{s_{0}}} \tau\right)^{\frac{1}{2}}\left(\frac{C}{\left(1-4 C\left\|u_{0}\right\|_{\left.B_{2,1}^{s_{0}} \tau\right)}\right.}+\frac{2 C\left\|u_{0}\right\|_{B_{2,1}^{s_{0}}}}{\left(1-4 C\left\|u_{0}\right\|_{\left.B_{2,1}^{s_{0}} \tau\right)^{2}}\right.}\right) d \tau \\
& =\frac{\left\|u_{0}\right\|_{B_{2,1}^{s_{0}}}}{\left(1-4 C\left\|u_{0}\right\|_{\left.B_{2,1}^{s_{0}} t\right)^{1 / 2}}\right.} \int_{0}^{t}\left(\frac{C}{\left(1-4 C\left\|u_{0}\right\|_{\left.B_{2,1}^{s_{0}} \tau\right)^{1 / 2}}\right.}+\frac{2 C\left\|u_{0}\right\|_{B_{2,1}^{s_{0}}}}{\left(1-4 C\left\|u_{0}\right\|_{B_{2,1}^{s_{0}}} \tau\right)^{3 / 2}}\right) d \tau \\
& =\frac{\left\|u_{0}\right\|_{B_{2,1}^{s_{0}}}}{\left(1-4 C\left\|u_{0}\right\|_{\left.B_{2,1}^{s_{0}} t\right)^{1 / 2}}\right.}\left(\frac{C t}{\left(1-4 C\left\|u_{0}\right\|_{\left.B_{2,1}^{s_{0}} \xi_{2}\right)^{1 / 2}}\right.}+\left.\left(1-4 C\left\|u_{0}\right\|_{B_{2,1}^{s_{0}} \tau}\right)^{-1 / 2}\right|_{0} ^{t}\right) \\
& \leq \frac{\left\|u_{0}\right\|_{B_{2,1}^{s_{0}}}}{\left(1-4 C\left\|u_{0}\right\|_{B_{2,1}^{s_{0}}} t\right)^{1 / 2}}\left(\frac{C T+1}{\left(1-4 C\left\|u_{0}\right\|_{\left.B_{2,1}^{s_{0}} t\right)^{1 / 2}}\right.}-1\right) \text {, }
\end{aligned}
$$


where the mean-value theorem for the integral has been employed with $0<\xi_{1}<t$. Inserting (4.9) and (4.10) into (4.5), we get that

$$
\left\|u^{(n+1)}\right\|_{B_{2,1}^{s_{0}}} \leq \frac{(1+C T)\left\|u_{0}\right\|_{B_{2,1}^{s_{0}}}}{1-4 C\left\|u_{0}\right\|_{B_{2,1}^{s_{0}} t}} \leq \frac{2\left\|u_{0}\right\|_{B_{2,1}^{s_{0}}}}{1-4 C\left\|u_{0}\right\|_{B_{2,1}^{s_{0}} t}} .
$$

Thus, $\left(u^{(n)}\right)_{n \in \mathbb{N}}$ is uniformly bounded in $C\left([0, T] ; B_{2,1}^{s_{0}}\right)$. Using equation (4.1), one can easily prove that $\left(\partial_{t} u^{(n)}\right)_{n \in \mathbb{N}}$ is uniformly bounded in $C\left([0, T] ; B_{2,1}^{s_{0}-1}\right)$. Consequently, $\left(u^{(n)}\right)_{n \in \mathbb{N}} \subset C\left([0, T] ; B_{2,1}^{s_{0}}\right) \cap C^{1}\left([0, T] ; B_{2,1}^{s_{0}-1}\right)$.

Third step: Convergence. We first show that $\left(u^{(n)}\right)_{n \in \mathbb{N}}$ is a Cauchy sequence in $C\left([0, T] ; B_{2, \infty}^{s_{0}-1}\right)$, then by using (2.1) we prove that $\left(u^{(n)}\right)_{n \in \mathbb{N}}$ is a Cauchy sequence in $C\left([0, T] ; B_{2,1}^{s_{0}-1}\right)$. For $(m, n) \in \mathbb{N}^{2}$, we have

$$
\begin{aligned}
& {\left[\partial_{t}+\left(1+u^{(n+m)}\right) \partial_{x}\right]\left(u^{(n+1+m)}-u^{(n+1)}\right)} \\
& =-\left(u^{(n+m)}-u^{(n)}\right) u_{x}^{(n+1)}+\partial_{x} P(D)\left[\left(u^{(n+m)}-u^{(n)}\right)+\left(\left(u^{(n+m)}\right)^{2}-\left(u^{(n)}\right)^{2}\right)\right] \\
& \quad+P(D)\left(\left[u^{(n+m)},\left(-\partial_{x}^{2}\right)^{\nu}\right] u_{x}^{(n+m)}-\left[u^{(n)},\left(-\partial_{x}^{2}\right)^{\nu}\right] u_{x}^{(n)}\right) \\
& \quad:=S_{1}(x, t)+S_{2}(x, t)+S_{3}(x, t) .
\end{aligned}
$$

We define

$$
w_{n, m}=\left\|\left(u^{(n+m)}-u^{(n)}\right)(t)\right\|_{B_{2, \infty}^{s_{0}-1}}
$$

and

$$
w_{n}(t)=\sup _{m \in \mathbb{N}} w_{n, m}(t)
$$

as well as

$$
\widetilde{w}(t)=\limsup _{n \rightarrow \infty} w_{n}(t) .
$$

We will show $\widetilde{w}(t)=0$, for $t \in[0, T]$. By (4.6), (4.7) and (4.9), we have that

$$
\left\|u^{(n)}(t)\right\|_{B_{2,1}^{s_{0}}} \leq 4\left\|u_{0}\right\|_{B_{2,1}^{s_{0}}}
$$

and

$$
e^{C \int_{0}^{t}\left\|\left(u^{(n)}\right)\left(t^{\prime}\right)\right\|_{B_{2,1}^{s_{0}}} d t^{\prime}} \leq 2
$$

Using (2)-(5) and (10) of Lemma 2.2 as well as (3.15)-(3.16) of Lemma3.4 and the above inequality, we obtain

$$
\begin{aligned}
& \left\|\left(u^{(n+m)}-u^{(n)}\right) u_{x}^{(n+1)}\right\|_{B_{2, \infty}^{s_{0}-1}} \\
& \leq C\left(\left\|u^{(n+m)}-u^{(n)}\right\|_{B_{2, \infty}^{s_{0}-1}}\left\|u_{x}^{(n+1)}\right\|_{L^{\infty}}+\left\|u_{x}^{(n+1)}\right\|_{B_{2, \infty}^{s_{0}-1}}\left\|u^{(n+m)}-u^{(n)}\right\|_{L^{\infty}}\right) \\
& \leq C\left\|u^{(n+m)}-u^{(n)}\right\|_{B_{2,1}^{s_{0}-1}}\left\|u^{(n+1)}\right\|_{B_{2,1}^{s_{0}},} \\
& \left\|\partial_{x} P(D)\left(u^{(n+m)}-u^{(n)}\right)\right\|_{B_{2, \infty}^{s_{0}-1}} \leq\left\|u^{(n+m)}-u^{(n)}\right\|_{B_{2, \infty}^{s_{0}-2 \nu}} \leq C\left\|u^{(n+m)}-u^{(n)}\right\|_{B_{2,1}^{s_{0}-1}}
\end{aligned}
$$




$$
\begin{aligned}
& \left\|\partial_{x} P(D)\left(\left(u^{(n+m)}\right)^{2}-\left(u^{(n)}\right)^{2}\right)\right\|_{B_{2, \infty}^{s_{0}-1}} \\
& \leq C\left\|\left(u^{(n+m)}\right)^{2}-\left(u^{(n)}\right)^{2}\right\|_{B_{2, \infty}^{s_{0}-2 \nu}} \\
& \leq C\left\|\left(u^{(n+m)}-u^{(n)}\right)\left(u^{(n+m)}+u^{(n)}\right)\right\|_{B_{2, \infty}^{s_{0}-1}} \\
& \leq C\left(\left\|u^{(n+m)}-u^{(n)}\right\|_{B_{2, \infty}^{s_{0}-1}}\left\|u^{(n+m)}+u^{(n)}\right\|_{L^{\infty}}+\left\|u^{(n+m)}+u^{(n)}\right\|_{B_{2, \infty}^{s_{0}-1}}\left\|u^{(n+m)}-u^{(n)}\right\|_{L^{\infty}}\right) \\
& \leq C\left\|u^{(n+m)}-u^{(n)}\right\|_{B_{2,1}^{s_{0}-1}}\left(\left\|u^{(n+m)}\right\|_{B_{2,1}^{s_{0}}}+\left\|u^{(n)}\right\|_{B_{2,1}^{s_{0}}}\right), \\
& \left\|P(D)\left(\left[u^{(n+m)},\left(-\partial_{x}^{2}\right)^{\nu}\right] u_{x}^{(n+m)}-\left[u^{(n)},\left(-\partial_{x}^{2}\right)^{\nu}\right] u_{x}^{(n)}\right)\right\|_{B_{2, \infty}^{s_{0}-1}} \\
& =\left\|P(D)\left(\left[\left(u^{(n+m)}-u^{(n)}\right),\left(-\partial_{x}^{2}\right)^{\nu}\right] u_{x}^{(n+m)}-\left[u^{(n)},\left(-\partial_{x}^{2}\right)^{\nu}\right]\left(u_{x}^{(n+m)}-u_{x}^{(n)}\right)\right)\right\|_{B_{2, \infty}^{s_{0}-1}} \\
& \leq\left\|\left[u^{(n+m)}-u^{(n)},\left(-\partial_{x}^{2}\right)^{\nu}\right] u_{x}^{(n+m)}\right\|_{B_{2, \infty}^{s_{0}-1-2 \nu}}+\left\|\left[u^{(n)},\left(-\partial_{x}^{2}\right)^{\nu}\right]\left(u_{x}^{(n+m)}-u_{x}^{(n)}\right)\right\|_{B_{2, \infty}^{s_{0}-1-2 \nu}} \\
& \leq C\left\|u^{(n+m)}-u^{(n)}\right\|_{B_{2,1}^{s_{0}-1}}\left\|u_{x}^{(n+m)}\right\|_{B_{2,1}^{s_{0}-1}}+C\left\|u^{(n)}\right\|_{B_{2,1}^{s_{0}}}\left\|u_{x}^{(n+m)}-u_{x}^{(n)}\right\|_{B_{2,1}^{s_{0}-2}} \\
& \leq C\left\|u^{(n+m)}-u^{(n)}\right\|_{B_{2,1}^{s_{0}-1}}\left(\left\|u^{(n+m)}\right\|_{B_{2,1}^{s_{0}}}+\left\|u^{(n)}\right\|_{\left.B_{2,1}^{s_{0}}\right)} .\right.
\end{aligned}
$$

We define

$$
M=24\left\|u_{0}\right\|_{B_{2,1}^{s_{0}}}+2,
$$

then we have from above inequalities and (4.16) that

$$
\begin{aligned}
& \left\|S_{1}(x, t)+S_{2}(x, t)+S_{3}(x, t)\right\|_{B_{2, \infty}^{s_{0}-1}} \\
& \leq C\left\|u^{(n+m)}-u^{(n)}\right\|_{B_{2,1}^{s_{0}-1}}\left(1+\left\|u^{(n+m)}\right\|_{B_{2,1}^{s_{0}}}+\left\|u^{(n)}\right\|_{B_{2,1}^{s_{0}}}+\left\|u^{(n+1)}\right\|_{B_{2,1}^{s_{0}}}\right) \\
& \leq C \frac{M}{2}\left\|u^{(n+m)}-u^{(n)}\right\|_{B_{2,1}^{s_{0}-1}} .
\end{aligned}
$$

Note that

$$
\begin{aligned}
\left\|u_{0}^{(n+1+m)}-u_{0}^{(n+1)}\right\|_{B_{2, \infty}^{s_{0}-1}} & =\left\|\left(S_{n+1+m} u_{0}-S_{n+1} u_{0}\right)\right\|_{B_{2, \infty}^{s_{0}-1}}=\left\|\sum_{q=n+1}^{n+m} \triangle_{q} u_{0}\right\|_{B_{2, \infty}^{s_{0}-1}} \\
& =\sup _{k \geq 1} 2^{\left(s_{0}-1\right) k}\left\|\triangle_{k}\left(\sum_{q=n+1}^{n+m} \triangle_{q} u_{0}\right)\right\|_{L^{2}} \\
& =\sup _{n+1 \leq k \leq n+m+1} 2^{-k} 2^{s_{0} k}\left\|\triangle_{k-1} \triangle_{k} u_{0}+\triangle_{k+1} \triangle_{k} u_{0}\right\|_{L^{2}} \\
& \leq \sup _{n \leq k \leq n+m} 2^{-k} 2^{s_{0} k}\left\|\triangle_{k} u_{0}\right\|_{L^{2}} \leq C 2^{-n}\left\|u_{0}\right\|_{B_{2,1}^{s_{0}}}
\end{aligned}
$$

Applying (2.2) of Lemma 2.3 and using (4.17)-(4.20), we have for $t \in[0, T]$,

$$
\begin{aligned}
& \left\|\left(u^{(n+1+m)}-u^{(n+1)}\right)(t)\right\|_{B_{2, \infty}^{s_{0}-1}} \\
& \leq e^{C \int_{0}^{t}\left\|u^{(n+m)}\left(t^{\prime}\right)\right\|_{B_{2, \infty}^{s_{0}-1}} d t^{\prime}}\left\|u_{0}^{(n+1+m)}-u_{0}^{(n+1)}\right\|_{B_{2, \infty}^{s_{0}-1}}
\end{aligned}
$$




$$
\begin{aligned}
& \quad+\int_{0}^{t} e^{C \int_{\tau}^{t}\left\|u^{(n+m)}\left(t^{\prime}\right)\right\|_{B_{2, \infty}^{s_{0}-1}} d t^{\prime}}\left\|S_{1}(x, t)+S_{2}(x, t)+S_{3}(x, t)\right\|_{B_{2, \infty}^{s_{0}-1}} d \tau \\
& \leq C M 2^{-n}+C M \int_{0}^{t}\left\|u^{(n+m)}-u^{(n)}\right\|_{B_{2,1}^{s_{0}-1}} d \tau .
\end{aligned}
$$

Combing (4.21), (4.13) and (11) of Lemma 2.2, we know that for $\forall(n, m) \in \mathbb{N}^{2}$

$$
\begin{aligned}
w_{n+1, m} & =\left\|u^{(n+1+m)}-u^{(n+1)}\right\|_{B_{2, \infty}^{s_{0}-1}} \\
& \leq C M\left(2^{-n}+\int_{0}^{t}\left\|\left(u^{(n+m)}-u^{(n)}\right)\right\|_{B_{2,1}^{s_{0}-1}} d \tau\right) \\
& \leq C M\left[2^{-n}+\int_{0}^{t}\left\|\left(u^{(n+m)}-u^{(n)}\right)\right\|_{B_{2, \infty}^{s_{0}-1}} \ln \left(e+\frac{\left\|\left(u^{(n+m)}-u^{(n)}\right)\right\|_{B_{2, \infty}^{s_{0}}}}{\left\|\left(u^{(n+m)}-u^{(n)}\right)\right\|_{B_{2, \infty}^{s_{0}-1}}}\right) d \tau\right] \\
& \leq C M\left[2^{-n}+\int_{0}^{t} w_{n, m}(\tau) \ln \left(e+\frac{M}{w_{n, m}(\tau)}\right) d \tau\right] .
\end{aligned}
$$

By (4.14) and 4.22), we have

$$
w_{n+1} \leq C M\left[2^{-n}+\int_{0}^{t} w_{n}(\tau) \ln \left(e+\frac{M}{w_{n}(\tau)}\right) d \tau\right] .
$$

Letting $n \rightarrow+\infty$ in 4.23 yields

$$
\widetilde{w}(t) \leq C M \int_{0}^{t} \widetilde{w}(\tau) \ln \left(e+\frac{M}{\widetilde{w}(\tau)}\right) d \tau
$$

Because for $x \in(0,1]$ and $\alpha>0$, we have

$$
\ln \left(e+\frac{\alpha}{x}\right) \leq \ln (e+\alpha)(1-\ln x) .
$$

Then the inequality (4.24) can be rewritten as

$$
\widetilde{w}(t) \leq C M \int_{0}^{t} \widetilde{w}(\tau) \ln (e+M)(1-\ln \widetilde{w}(\tau)) d \tau
$$

provided that $\widetilde{w}(t) \leq 1$ on $[0, T]$. Using a Gronwall type argument (see e.g. Lemma 5.2.1 in [7]) yields $\widetilde{w}(t)=0$ for $t \in[0, T]$.

Now we claim that $\left(u^{(n)}\right)_{n \in \mathbb{N}}$ is a Cauchy sequence in $C\left([0, T] ; B_{2,1}^{s_{0}-1}\right)$. Using (2.1) of Lemma 2.2, we have that

$$
\begin{aligned}
& \left\|\left(u^{(n+1+m)}-u^{(n+1)}\right)(t)\right\|_{B_{2,1}^{s_{0}-1}} \\
& \leq C(\theta)\left\|\left(u^{(n+1+m)}-u^{(n+1)}\right)(t)\right\|_{B_{2, \infty}^{s_{0}-1}}^{\theta}\left\|\left(u^{(n+1+m)}-u^{(n+1)}\right)(t)\right\|_{B_{2, \infty}^{s_{0}}}^{1-\theta} \\
& \leq C(\theta)\left\|\left(u^{(n+1+m)}-u^{(n+1)}\right)(t)\right\|_{B_{2, \infty}^{s_{0}-1}}^{\theta}\left(\left\|u^{(n+1+m)}(t)\right\|_{B_{2,1}^{s_{0}}}^{(n+\|}+u^{(n+1)}(t) \|_{B_{2,1}^{s_{0}}}\right)^{1-\theta} \\
& \leq C(\theta)(C M)^{1-\theta}\left\|\left(u^{(n+1+m)}-u^{(n+1)}\right)(t)\right\|_{B_{2, \infty}^{s_{0}-1}}^{\theta} \\
& =C(\theta)(C M)^{1-\theta} w_{n+1, m}^{\theta}(t) .
\end{aligned}
$$


For $\forall t \in[0, T], m \in \mathbb{N}$, we get from (4.27) that

$$
\limsup _{n \rightarrow \infty}\left\|\left(u^{(n+1+m)}-u^{(n+1)}\right)(t)\right\|_{B_{2,1}^{s_{0}-1}}=0 .
$$

Thus, $\left(u^{(n)}\right)_{n \in \mathbb{N}}$ is a Cauchy sequence in $C\left([0, T] ; B_{2,1}^{s_{0}-1}\right)$, whence $\left(u^{(n)}\right)_{n \in \mathbb{N}}$ converges to some limit $u \in C\left([0, T] ; B_{2,1}^{s_{0}-1}\right)$.

Fourth step: Existence and continuity of solution in $E_{2,1}^{s_{0}}(T)$. Now we have to check that $u$ belongs to $E_{2,1}^{s_{0}}(T)$ and satisfies (1.5). Since $\left(u^{(n)}\right)_{n \in \mathbb{N}}$ is uniformly bounded in $L^{\infty}\left([0, T] ; B_{2,1}^{s_{0}}\right)$. From (8) of Lemma 2.2, we have that $u \in L^{\infty}\left([0, T] ; B_{2,1}^{s_{0}}\right)$. From (1.5), we can easily prove that $u_{t} \in L^{\infty}\left([0, T] ; B_{2,1}^{s_{0}-1}\right)$. It is easily checked that $u$ is indeed a solution to (1.5) by passing to the limit in (4.1). Using similar proof to [15], we can obtain that $u \in E_{2,1}^{s_{0}}(T)$.

Fifth step: Uniqueness. Uniqueness is a corollary of the following result.

Proposition 4.1. Let $v$, $u$ be solutions to the problem (1.5) with initial data $v_{0}, u_{0}$, respectively. Let $w(t):=v-u$. Obviously, $w(0):=v_{0}-u_{0}$. There exists a constant $C$ such that if for some $T_{\star} \leq T$

$$
\sup _{t \in\left[0, T_{\star}\right]}\left(e^{-C \int_{0}^{t}\|u(\tau)\|_{B_{2, \infty}^{s_{0}-1}} d \tau}\|w(t)\|_{B_{2, \infty}^{s_{0}-1}}\right) \leq 1,
$$

then the following inequality holds true for $t \in\left[0, T_{\star}\right]$ :

$$
\|w(t)\|_{B_{2, \infty}^{s_{0}-1}} \leq e^{\left(1+C \int_{0}^{t}\|u(\tau)\|_{B_{2, \infty}^{s_{0}-1}} d \tau\right)}\left(\frac{\|w(0)\|_{B_{2, \infty}^{s_{0}-1}}}{e}\right)^{\exp [-C t Z \ln (e+Z)]},
$$

where $Z$ is defined as

$$
Z=4\left\|u_{0}\right\|_{B_{2,1}^{s_{0}}}+4\left\|v_{0}\right\|_{B_{2,1}^{s_{0}}}+1
$$

Furthermore, if

$$
\|w(0)\|_{B_{2, \infty}^{s_{0}-1}} \leq e^{1-\exp [C T Z \ln (e+Z)]},
$$

then (4.28) is valid on $[0, T]$. In particular, when $w(0)=0$, then $u(x, t)=v(x, t)$.

Proof. Obviously, $w$ solves the following Cauchy problem for the transport equation:

$$
\begin{aligned}
& w_{t}+(1+u) w_{x} \\
& =-w v_{x}+\partial_{x} P(D)(w+w(v+u))+P(D)\left(\left[w,\left(-\partial_{x}^{2}\right)^{\nu}\right] v_{x}-\left[u,\left(-\partial_{x}^{2}\right)^{\nu}\right] w_{x}\right) \\
& :=\widetilde{S}_{1}(x, t)+\widetilde{S}_{2}(x, t)+\widetilde{S}_{3}(x, t) .
\end{aligned}
$$

Using (2.2) of Lemma 2.3 and (4.30), we have

$$
\begin{aligned}
& \|w(t)\|_{B_{2, \infty}^{s_{0}-1}} \leq\|w(0)\|_{B_{2, \infty}^{s_{0}-1}} e^{C \int_{0}^{t}\|u(\tau)\|_{B_{2, \infty}^{s_{0}-1}} d \tau} \\
& +\int_{0}^{t} e^{C \int_{\tau}^{t}\left\|u\left(t^{\prime}\right)\right\|_{B_{2, \infty}^{s_{0}-1}} d t^{\prime}}\left\|\widetilde{S}_{1}(x, t)+\widetilde{S}_{2}(x, t)+\widetilde{S}_{3}(x, t)\right\|_{B_{2, \infty}^{s_{0}-1}}, d \tau .
\end{aligned}
$$


Following the proof of 4.19), we obtain

$$
\left\|\widetilde{S}_{1}(x, t)+\widetilde{S}_{2}(x, t)+\widetilde{S}_{3}(x, t)\right\|_{B_{2, \infty}^{s_{0}-1}} \leq C Z\|w\|_{B_{2,1}^{s_{0}-1}}
$$

Inserting (4.32) into (4.31) yields

$$
\begin{aligned}
& \|w(t)\|_{B_{2, \infty}^{s_{0}-1}} \\
& \leq\|w(0)\|_{B_{2, \infty}^{s_{0}-1}} e^{C \int_{0}^{t}\|u(\tau)\|_{B_{2, \infty}^{s_{0}-1}} d \tau}+C Z \int_{0}^{t} e^{C \int_{\tau}^{t}\left\|u\left(t^{\prime}\right)\right\|_{B_{2, \infty}^{s_{0}-1}} d t^{\prime}}\|w\|_{B_{2,1}^{s_{0}-1}} d \tau \\
& \leq\|w(0)\|_{B_{2, \infty}^{s_{0}-1}} e^{C \int_{0}^{t}\|u(\tau)\|_{B_{2, \infty}^{s_{0}-1}} d \tau}+C Z \int_{0}^{t} e^{C \int_{\tau}^{t}\left\|u\left(t^{\prime}\right)\right\|_{B_{2, \infty}^{s_{0}-1}} d t^{\prime}}\|w\|_{B_{2, \infty}^{s_{0}-1}} \ln \left(e+\frac{\|w\|_{B_{2, \infty}^{s_{0}}}}{\|w\|_{B_{2, \infty}^{s_{0}-1}}} d \tau\right. \\
& \leq C Z \int_{0}^{t} e^{C \int_{\tau}^{t}\left\|u\left(t^{\prime}\right)\right\|_{B_{2, \infty}^{s_{0}-1}} d t^{\prime}}\|w\|_{B_{2, \infty}^{s_{0}-1}} \ln \left(e+\frac{Z}{e^{-C \int_{0}^{\tau}\left\|u\left(t^{\prime}\right)\right\|_{B_{2, \infty}}^{s_{0}-1} d t^{\prime}}\|w\|_{B_{2, \infty}^{s_{0}-1}}}\right) d \tau \\
& \quad+\|w(0)\|_{B_{2, \infty}^{s_{0}-1}} e^{C \int_{0}^{t}\|u(\tau)\|_{B_{2, \infty}^{s_{0}-1}} d \tau} .
\end{aligned}
$$

Denote

$$
W(t)=e^{-C \int_{0}^{t}\|u(\tau)\|_{B_{2, \infty}^{s_{0}-1} d \tau}}\|w\|_{B_{2, \infty}^{s_{0}-1}}
$$

Inequality (4.33) can be rewritten as

$$
W(t) \leq W(0)+C Z \int_{0}^{t} W(\tau) \ln \left(e+\frac{Z}{W(\tau)}\right) d \tau
$$

In light of the hypothesis and using a Gronwall type argument [39] yields

$$
\frac{W(t)}{e} \leq\left(\frac{W(0)}{e}\right)^{\exp [-C t Z \ln (e+Z)]}
$$

implying the desired result. (4.29) implies that (4.28) is valid with $T_{\star}=T$.

Sixth step: Continuity with respect to the initial data in $B_{2,1}^{s_{0}}$.

Proposition 4.2. For any $u_{0} \in B_{2,1}^{s_{0}}$, there exist a $T>0$ and a neighborhood $V$ of $u_{0}$ in $B_{2,1}^{s_{0}}$ such that the map

$$
\Phi:\left\{\begin{array}{l}
V \subset B_{2,1}^{s_{0}} \rightarrow C\left([0, T] ; B_{2,1}^{s_{0}}\right) \\
v_{0} \rightarrow v \text { solution to } 1.5 \text { with initial datum } v_{0}
\end{array}\right.
$$

is continuous.

Proof. Motivated by [16], we prove Proposition 4.2 by using Lemma 2.5.

First step: Continuity in $C\left([0, T] ; B_{2,1}^{s_{0}-1}\right)$. For $u_{0} \in B_{2,1}^{s_{0}}$ and $r>0$, we claim that there exist a $T>0$ and a $M>0$ such that for any $u_{0}^{\prime} \in B_{2,1}^{s_{0}}$ with $\left\|u_{0}-u_{0}^{\prime}\right\|_{B_{2,1}^{s_{0}}} \leq r$, the solution $u^{\prime}=\Phi\left(u_{0}^{\prime}\right)$ of (1.5) associated with $u_{0}^{\prime}$ belongs to $C\left([0, T] ; B_{2,1}^{s_{0}}\right)$ and satisfies

$$
\left\|u^{\prime}\right\|_{L^{\infty}\left(0, T ; B_{2,1}^{s_{0}}\right)} \leq M
$$


Indeed, from

$$
\left\|u^{\prime}\right\|_{B_{2,1}^{s_{0}}} \leq \frac{2\left\|u_{0}^{\prime}\right\|_{B_{2,1}^{s_{0}}}}{1-4 C\left\|u_{0}^{\prime}\right\|_{B_{2,1}^{s_{0}} t}}
$$

we know that $T<\frac{1}{4 C\left\|u_{0}^{\prime}\right\|_{B_{2,1}^{s_{0}}}}$. Thus we can choose

$$
T=\frac{1}{8 C\left(\left(\left\|u_{0}\right\|_{B_{2,1}^{s_{0}}}+r\right)+r\right)}, \quad M=4\left\|u_{0}\right\|_{B_{2,1}^{s_{0}}}+4 r .
$$

Then

$$
T \leq \frac{1}{8 C\left(\left\|u_{0}^{\prime}\right\|_{B_{2,1}^{s_{0}}}+r\right)}
$$

and

$$
\left\|u^{\prime}\right\|_{B_{2,1}^{s_{0}}} \leq \frac{2\left\|u_{0}^{\prime}\right\|_{B_{2,1}^{s_{0}}}}{1-\frac{\left\|u_{0}^{\prime}\right\|_{B_{2,1}^{s_{0}}}}{2\left(\left\|u_{0}^{\prime}\right\|_{\left.B_{2,1}^{s_{0}}+r\right)}\right.}} \leq 4\left\|u_{0}^{\prime}\right\|_{B_{2,1}^{s_{0}}} \leq M .
$$

Combining the above uniform bounds with Proposition 4.1, we infer that

$$
\left\|\Phi\left(u_{0}^{\prime}\right)-\Phi\left(u_{0}\right)\right\|_{L^{\infty}\left(0, T ; B_{2, \infty}^{s_{0}-1}\right)} \leq e^{(1+C M T)}\left(\frac{\left\|u_{0}^{\prime}-u_{0}\right\|_{B_{2, \infty}^{s_{0}-1}}}{e}\right)^{\exp [-C T Z \ln (e+Z)]}
$$

provided that

$$
\left\|u_{0}^{\prime}-u_{0}\right\|_{B_{2, \infty}^{s_{0}-1}} \leq e^{1-\exp [C T Z \ln (e+Z)]} .
$$

In view of the uniform bounds in $C\left([0, T] ; B_{2,1}^{s_{0}}\right)$ and an interpolation argument, we infer the map $\Phi$ is continuous from $B_{2,1}^{s_{0}}$ into $C\left([0, T] ; B_{2,1}^{s_{0}-1}\right)$.

Second step: Continuity in $C\left([0, T] ; B_{2,1}^{s_{0}}\right)$. Let $u_{0}^{(\infty)} \in B_{2,1}^{s_{0}}$ and $\left(u_{0}^{(n)}\right)_{n \in \mathbb{N}}$ tend to $u_{0}^{(\infty)}$ in $B_{2,1}^{s_{0}}$. We denote by $u^{(n)}$ the solution with the initial data $u_{0}^{(n)}$. From the first step, we can find $T, M>0$ such that for all $n \in \mathbb{N}, u^{(n)}$ is defined on $[0, T]$ and

$$
\sup _{n \in \overline{\mathbb{N}}}\left\|u^{(n)}\right\|_{L^{\infty}\left(0, T ; B_{2,1}^{\left.s_{0}\right)}\right.} \leq M
$$

Thanks to step one, proving that $u^{(n)}$ tends to $u^{(\infty)}$ in $C\left([0, T] ; B_{2,1}^{s_{0}}\right)$ amounts to proving that $v^{(n)}=\partial_{x} u^{(n)}$ tends to $v^{(\infty)}=\partial_{x} u^{(\infty)}$ in $C\left([0, T] ; B_{2,1}^{s_{0}-1}\right)$. Notice that $v^{(n)}$ solves the following linear transport equations

$$
\left\{\begin{array}{l}
\partial_{t} v^{(n)}+\left(1+u^{(n)}\right) \partial_{x} v^{(n)}=\widetilde{f}^{(n)} \\
\left.v^{(n)}\right|_{t=0}=\partial_{x} u_{0}^{(n)}
\end{array}\right.
$$

with

$$
\widetilde{f}^{(n)}=-\left(u_{x}^{(n)}\right)^{2}+\partial_{x}^{2} P(D) f_{1}\left(u^{(n)}\right)+\partial_{x} P(D) f_{2}\left(u^{(n)}, u_{x}^{(n)}\right) .
$$


Following the method in [31], we decompose $v^{(n)}=v_{1}^{(n)}+v_{2}^{(n)}$ with

$$
\left\{\begin{array}{l}
\partial_{t} v_{1}^{(n)}+\left(1+u^{(n)}\right) \partial_{x} v_{1}^{(n)}=\widetilde{f}^{(n)}-\widetilde{f}^{(\infty)}, \\
\left.v_{1}^{(n)}\right|_{t=0}=\partial_{x} u_{0}^{(n)}-\partial_{x} u_{0}^{(\infty)}
\end{array}\right.
$$

and

$$
\left\{\begin{array}{l}
\partial_{t} v_{2}^{(n)}+\left(1+u^{(n)}\right) \partial_{x} v_{2}^{(n)}=\widetilde{f}^{(\infty)}, \\
\left.v_{2}^{(n)}\right|_{t=0}=\partial_{x} u_{0}^{(\infty)}
\end{array}\right.
$$

On the other hand, we have

$$
\begin{aligned}
\left\|\left(u_{x}^{(n)}\right)^{2}\right\|_{B_{2,1}^{s_{0}-1}} \leq\left\|u_{x}^{(n)}\right\|_{B_{2,1}^{s_{0}-1}}^{2} & \leq\left\|u^{(n)}\right\|_{B_{2,1}^{s_{0}}}^{2} \\
\left\|\partial_{x}^{2} P(D) f_{1}\left(u^{(n)}\right)\right\|_{B_{2,1}^{s_{0}-1}} & \leq\left\|u^{(n)}+\left(u^{(n)}\right)^{2}\right\|_{B_{2,1}^{s_{0}+1-2 \nu}} \\
& \leq\left\|u^{(n)}+\left(u^{(n)}\right)^{2}\right\|_{B_{2,1}^{s_{0}-1}} \leq\left\|u^{(n)}\right\|_{B_{2,1}^{s_{0}}}+\left\|u^{(n)}\right\|_{B_{2,1}^{s_{0}}}^{2}, \\
\left\|\partial_{x} P(D) f_{2}\left(u^{(n)}, u_{x}^{(n)}\right)\right\|_{B_{2,1}^{s_{0}-1}} & \leq\left\|\left[u^{(n)},\left(-\partial_{x}^{2}\right)^{\nu}\right] u_{x}^{(n)}\right\|_{B_{2,1}^{s_{0}-2 \nu}} \\
& \leq\left\|u^{(n)}\right\|_{B_{2,1}^{s_{0}}}\left\|u_{x}^{(n)}\right\|_{B_{2,1}^{s_{0}-1}} \leq\left\|u^{(n)}\right\|_{B_{2,1}^{s_{0}}}^{2},
\end{aligned}
$$

and thus $\left(\widetilde{f}^{(n)}\right)_{n \in \mathbb{N}}$ is uniformly bounded in $C\left([0, T] ; B_{2,1}^{s_{0}}\right)$. A similar argument yields the following inequalities

$$
\begin{aligned}
& \left\|\widetilde{f}^{(n)}-\widetilde{f}^{(\infty)}\right\|_{B_{2,1}^{s_{0}-1}} \\
& \leq C\left(1+\left\|u^{(n)}\right\|_{B_{2,1}^{s_{0}}}+\left\|u^{(\infty)}\right\|_{B_{2,1}^{s_{0}}}\right)\left(\left\|u^{(n)}-u^{(\infty)}\right\|_{B_{2,1}^{s_{0}}}+\left\|u_{x}^{(n)}-u_{x}^{(\infty)}\right\|_{B_{2,1}^{s_{0}-1}}\right) .
\end{aligned}
$$

Applying Lemma 2.3, one can deduce that

$$
\begin{aligned}
& \left\|v_{1}^{(n)}(t)\right\|_{B_{2,1}^{s_{0}-1}} \leq e^{C \int_{0}^{t}\|u(\tau)\|_{B_{2,1}^{s_{0}} d \tau}}\left\|\partial_{x} u_{0}^{(n)}-\partial_{x} u_{0}^{(\infty)}\right\|_{B_{2,1}^{s_{0}-1}} \\
& +C \int_{0}^{t} e^{C \int_{\tau}^{t}\left\|u_{x}\left(\tau^{\prime}\right)\right\|_{B_{2,1}^{s_{0}-1}} d \tau^{\prime}}\left\|\widetilde{f}^{(n)}-\widetilde{f}^{(\infty)}\right\|_{B_{2,1}^{s_{0}-1}} d \tau \\
& \leq e^{C \int_{0}^{t}\|u(\tau)\|_{B_{2,1}^{s_{0}} d \tau}}\left\|\partial_{x} u_{0}^{(n)}-\partial_{x} u_{0}^{(\infty)}\right\|_{B_{2,1}^{s_{0}-1}} \\
& +C \int_{0}^{t} e^{C \int_{\tau}^{t}\left\|u_{x}\left(\tau^{\prime}\right)\right\|_{B_{2,1}^{s_{0}-1} d \tau^{\prime}}}\left(\left\|u^{(n)}-u^{(\infty)}\right\|_{B_{2,1}^{s_{0}-1}}+\left\|u_{x}^{(n)}-u_{x}^{(\infty)}\right\|_{B_{2,1}^{s_{0}-1}}\right) \\
& \times\left(1+\left\|u^{(n)}\right\|_{B_{2,1}^{s_{0}}}+\left\|u^{(\infty)}\right\|_{B_{2,1}^{s_{0}}}\right) d \tau .
\end{aligned}
$$

Applying similar arguments as in [16] on P. 441 to [4.35), we have

$$
\partial_{x} u^{(n)} \rightarrow \partial_{x} u^{(\infty)} \quad \text { in } B_{2,1}^{s_{0}-1} .
$$

We have completed the proof of Proposition 4.2.

Summing up the above six steps, we get Theorem 1.1 


\section{Proof of Theorem 1.2}

In this section, we are devoted to establishing the existence and uniqueness of analytic solutions to the system (1.5) on the line $\mathbb{R}$.

The proof of Theorem 1.2 needs a suitable scale of Banach spaces as follows. For any $s>0$, we set

$$
E_{s}=\left\{u \in C^{\infty}(\mathbb{R}):\|u\|_{s}=\sup _{k \in \mathbb{N}_{0}} \frac{s^{k}\left\|\partial_{x}^{k} u\right\|_{B_{2,1}^{s_{0}}}}{k ! /(k+1)^{2}}<\infty\right\},
$$

where $\mathbb{N}_{0}$ is the set of nonnegative integers. We take note that the above space is similar to the one introduced in [26], where $B_{2,1}^{s_{0}}$ is replaced by $H^{2}$. It is easy to verify that $E_{s}$ equipped with the norm $\|\cdot\| \cdot \|$ is a Banach space and that for any $0<s^{\prime}<s, E_{s}$ is continuously embedded in $E_{s^{\prime}}$ with

$$
\|u\|_{s^{\prime}} \leq\|u\|_{s} .
$$

By this definition, one can easily get that $u$ in $E_{s}$ is a real analytic function on $\mathbb{R}$ and what is crucial for our purposes is the fact that each $E_{s}$ forms an algebra under pointwise multiplication of functions.

Lemma 5.1. (1) Let $0<s<1$. There is a constant $C>0$, independent of $s$, such that for any $u$ and $v$ in $E_{s}$ we have

$$
\|u v\|_{s} \leq C\|u\|\left\|_{s}\right\| v \|_{s} .
$$

(2) There is a constant $C>0$ such that for any $0<s^{\prime}<s<1$, we have

$$
\begin{gathered}
\left\|\partial_{x} u\right\|_{s^{\prime}} \leq \frac{C}{s-s^{\prime}}\|u\|_{s}, \\
\|P(D) u\|_{s} \leq C\|u\|_{s}, \quad\left\|\partial_{x} P(D) u\right\|_{s} \leq C\|u\|\left\|_{s}, \quad\right\| \partial_{x}^{2} P(D) u\|\|_{s} \leq C\|\| u \|_{s},
\end{gathered}
$$

and

$$
\left\|P(D)\left[u,\left(-\partial_{x}^{2}\right)^{\nu}\right] v_{x}\right\|_{s} \leq C\|\| u\|\|_{s}\|v\|_{s} .
$$

Proof. The properties (5.3) and (5.4) follow directly from analogous ones found in [26] just by replacing $H^{2}$ with $B_{2,1}^{s_{0}}$ and we then prove (5.5). Since

$$
\left\|\partial_{x}^{k} \partial_{x}^{2} P(D) u\right\|_{B_{2,1}^{s_{0}}} \leq C\left\|\partial_{x}^{k} u\right\|_{B_{2,1}^{s_{0}-2 \nu+2}} \leq C\left\|\partial_{x}^{k} u\right\|_{B_{2,1}^{s_{0}}},
$$

then it follows that

$$
\begin{aligned}
\left\|\partial_{x}^{2} P(D) u\right\|_{s} & =\sup _{k \in \mathbb{N}_{0}} \frac{s^{k}\left\|\partial_{x}^{k} \partial_{x}^{2} P(D) u\right\|_{B_{2,1}^{s_{0}}}}{k ! /(k+1)^{2}} \\
& \leq \sup _{k \in \mathbb{N}_{0}} \frac{s^{k}\left\|\partial^{k} u\right\|_{B_{2,1}^{s_{0}}}}{k ! /(k+1)^{2}}=\|u\|_{s} .
\end{aligned}
$$


The other estimates in (5.5) can be obtained similarly as above. Now we prove (5.6). As

$$
\begin{aligned}
& \left\|\partial_{x}^{k} P(D)\left[u,\left(-\partial_{x}^{2}\right)^{\nu}\right] v_{x}\right\|_{B_{2,1}^{s_{0}}} \\
& \leq\left\|\partial_{x}^{k}\left(\left[u,\left(-\partial_{x}^{2}\right)^{\nu}\right] v_{x}\right)\right\|_{B_{2,1}^{s_{0}-2 \nu}} \\
& =\sum_{l=0}^{k}\left(\begin{array}{c}
k \\
l
\end{array}\right)\left\|\left[\partial_{x}^{k-l} u,\left(-\partial_{x}^{2}\right)^{\nu}\right]\left(\partial_{x}^{l} v\right)_{x}\right\|_{B_{2,1}^{s_{0}-2 \nu}} \\
& \leq C \sum_{l=0}^{k}\left(\begin{array}{c}
k \\
l
\end{array}\right)\left\|\partial_{x}^{k-l} u\right\|_{B_{2,1}^{s_{0}}}\left\|\partial_{x}^{l} v\right\|_{B_{2,1}^{s_{0}}} \\
& =C\left\|\partial_{x}^{k} u\right\|_{B_{2,1}^{s_{0}}}\|v\|_{B_{2,1}^{s_{0}}}+C \sum_{l=1}^{k}\left(\begin{array}{c}
k \\
l
\end{array}\right)\left\|\partial_{x}^{k-l} u\right\|_{B_{2,1}^{s_{0}}}\left\|\partial_{x}^{l} u\right\|_{B_{2,1}^{s_{0}}}
\end{aligned}
$$

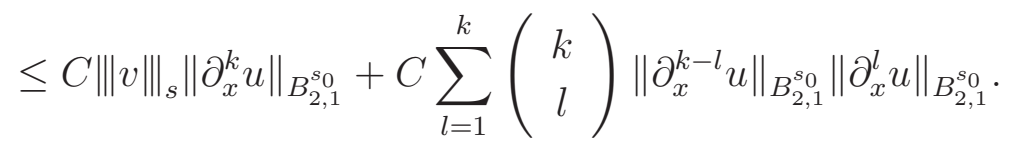

Then proceeding a similar argument as the proof of Lemma 2.1 in [38], we complete the proof of Lemma 5.1

Theorem 5.1. ([6]) Let $\left\{X_{s}\right\}_{0<s<1}$ be a scale of decreasing Banach spaces, namely for any $s^{\prime}<s$ we have $X_{s} \subset X_{s^{\prime}}$ and $\|\cdot \cdot\|_{s^{\prime}} \leq\|\cdot\| \|_{s}$. Consider the Cauchy problem

$$
\left\{\begin{array}{l}
\frac{d u}{d t}=F(t, u(t)) \\
u(0)=0
\end{array}\right.
$$

Let $T, R$ and $C$ be positive constants and assume that $F$ satisfies the following conditions:

(1) If for $0<s^{\prime}<s<1$ the function $t \mapsto u(t)$ is real analytic in $|t|<T$ and continuous on $|t| \leq T$ with values in $X_{s}$ and

$$
\sup _{|t| \leq T}\|u \mid\|_{s}<R
$$

then $t \mapsto F(t, u(t))$ is a real analytic function on $|t|<T$ with values in $X_{s^{\prime}}$.

(2) For any $0<s^{\prime}<s<1$ and any $u, v \in X_{s}$ with $\|u\|_{s}<R$, $\|v\|_{s}<R$,

$$
\sup _{|t| \leq T}\|F(t, u)-F(t, v)\|_{s^{\prime}} \leq \frac{C}{s-s^{\prime}}\|u-v\|_{s} .
$$

(3) There exists $M>0$ such that for any $0<s<1$

$$
\sup _{|t| \leq T}\|F(t, 0)\|_{s} \leq \frac{M}{1-s} .
$$

Then there exists a $T_{0} \in(0, T)$ and a unique function $u(t)$, which for every $0<s<1$ is real analytic in $|t|<(1-s) T_{0}$ with values in $X_{s}$, and is a solution to the Cauchy problem (5.7). 
To prove Theorem 1.2, we need to show that all three conditions of the abstract version of the Cauchy-Kowalevski theorem (Theorem 5.1) hold for system (1.5) on the scale $\left\{X_{s}\right\}_{0<s<1}$. To this end, we restate the Cauchy problem (1.5) in a more convenient form. Let $u_{1}=u, u_{2}=u_{x}$, then the problem (1.5) is transformed in a system for $u_{1}$ and $u_{2}$.

$$
\left\{\begin{array}{l}
\partial_{t} u_{1}=-u_{2}-\frac{1}{2} \partial_{x}\left(u_{1}^{2}\right)+\partial_{x} P(D) f_{1}\left(u_{1}\right)+P(D) f_{2}\left(u_{1}, u_{2}\right)=F_{1}\left(u_{1}, u_{2}\right), \\
\begin{array}{rl}
\partial_{t} u_{2} & =-\partial_{x}\left(u_{2}+u_{1} u_{2}\right)+\partial_{x}^{2} P(D) f_{1}\left(u_{1}\right)+\partial_{x} P(D) f_{2}\left(u_{1}, u_{2}\right) \\
& =F_{2}\left(u_{1}, u_{2}\right)
\end{array} \\
u_{1}(x, 0)=u_{0}(x), u_{2}(x, 0)=u_{0}^{\prime}(x) .
\end{array}\right.
$$

Proof of Theorem 1.2 Let $u=\left(u_{1}, u_{2}\right), F=\left(F_{1}, F_{2}\right)$ in (5.11) and $X_{s}$ be a scale of decreasing Banach spaces defined as $X_{s}=E_{s} \times E_{s}$. Since the map $F\left(u_{1}, u_{2}\right)$ does not depend on $t$ explicitly, we just need to verify the first two conditions of Theorem 5.1 .

Obviously, $t \mapsto F(t, u(t))=\left(F_{1}\left(u_{1}, u_{2}\right), F_{2}\left(u_{1}, u_{2}\right)\right)$ is real analytic if $t \mapsto u_{1}(t)$ and $t \mapsto u_{2}(t)$ are both real analytic. Hence, the verification of the first condition of the abstract theorem needs only to show that for $s^{\prime}<s, F_{1}\left(u_{1}, u_{2}\right)$ and $F_{2}\left(u_{1}, u_{2}\right)$ are in $E_{s^{\prime}}$ for $u_{1}, u_{2} \in E_{s}$. By Lemma5.1, we can get the estimates of $F_{1}$ and $F_{2}$ as

$$
\begin{aligned}
\left\|F_{1}\left(u_{1}, u_{2}\right)\right\| \|_{s^{\prime}} & =\left\|-u_{2}-\frac{1}{2} \partial_{x}\left(u_{1}^{2}\right)+\partial_{x} P(D) f_{1}\left(u_{1}\right)+P(D) f_{2}\left(u_{1}, u_{2}\right)\right\| \|_{s^{\prime}} \\
& \leq \frac{C}{s-s^{\prime}}\left(\left\|u_{1}\right\|\left\|_{s}+\right\| u_{1} \|_{s}^{2}\right)+C\left(\left\|u_{1}\right\|_{s}+\left\|u_{1}\right\|_{s}^{2}\right),
\end{aligned}
$$

and

$$
\begin{aligned}
& \left\|F_{2}\left(u_{1}, u_{2}\right)\right\| \|_{s^{\prime}} \\
& =\|\|-\partial_{x}\left(u_{2}+u_{1} u_{2}\right)+\partial_{x}^{2} P(D) f_{1}\left(u_{1}\right)+\partial_{x} P(D) f_{2}\left(u_{1}, u_{2}\right)\|\|_{s^{\prime}} \\
& \leq \frac{C}{s-s^{\prime}}\left(\left\|u_{2}\right\|_{s}+\left\|u_{1}\right\|\left\|_{s}\right\| u_{2}\left\|_{s}+\right\| u_{1} \|_{s}^{2}\right)+C\left(\left\|u_{1}\right\|_{s}+\left\|u_{1}\right\|_{s}^{2}\right) .
\end{aligned}
$$

We proceed to verify the second condition of the abstract theorem. Employing the triangle inequality and Lemma 5.1, we have

$$
\begin{aligned}
& \left\|F_{1}\left(u_{1}, u_{2}\right)-F_{1}\left(v_{1}, v_{2}\right)\right\| \|_{s^{\prime}} \\
& \leq\left\|\left(v_{2}-u_{2}\right)+\frac{1}{2} \partial_{x}\left(v_{1}^{2}-u_{1}^{2}\right)\right\| \|_{s^{\prime}} \\
& +\left\|\partial_{x} P(D)\left(f_{1}\left(u_{1}\right)-f_{1}\left(v_{1}\right)\right)+P(D)\left(f_{2}\left(u_{1}, u_{2}\right)-f_{2}\left(v_{1}, v_{2}\right)\right)\right\|_{s^{\prime}} \\
& \leq C\|\| u_{2}-v_{2}\left\|_{s}+\frac{C}{s-s^{\prime}}\right\| u_{1}-v_{1}\|\|_{s}\left\|u_{1}+v_{1}\right\|_{s} \\
& +\left\|\partial_{x} P(D)\left(\left(u_{1}-v_{1}\right)+\left(u_{1}-v_{1}\right)\left(u_{1}+v_{1}\right)\right)\right\|_{s^{\prime}} \\
& +\|\| P(D)\left(\left[u_{1}-v_{1},\left(-\partial_{x}^{2}\right)^{\nu}\right] \partial_{x} u_{1}-\left[v_{1},\left(-\partial_{x}^{2}\right)^{\nu}\right]\left(v_{1}-u_{1}\right)_{x}\right)\|\|_{s^{\prime}} \\
& \leq C\left\|u_{2}-v_{2}\right\|_{s}+\frac{C}{s-s^{\prime}}\left\|u_{1}-v_{1}\right\|\left\|_{s}\left(\left\|u_{1}\right\|_{s}+\left\|v_{1}\right\| \|_{s}\right)+C\right\| u_{1}-v_{1}\|\|_{s}
\end{aligned}
$$




$$
\begin{aligned}
& +C\left\|u_{1}-v_{1}\right\| \|_{s}\left(\left\|u_{1}\right\|_{s}+\left\|\left|v_{1}\right|\right\|_{s}\right) \\
& \leq \frac{C}{s-s^{\prime}}\|u-v\| \|_{X_{s}} .
\end{aligned}
$$

Similarly, we can show that

$$
\left\|F_{2}\left(u_{1}, u_{2}\right)-F_{2}\left(v_{1}, v_{2}\right)\right\|_{s^{\prime}} \leq \frac{C}{s-s^{\prime}}\|u-v\|_{X_{s}}
$$

holds. The conditions (1)-(3) are now easily verified once our system (5.11) is transformed into a new system with zero initial data as in (5.7). This completes the proof of Theorem 1.2.

Acknowledgements. The work of Fan is supported by a NSFC Grant No. 11701155. The work of Gao is partially supported by the NSFC grant No. 11531006, and the Jiangsu Center for Collaborative Innovation in Geographical Information Resource and Applications. The work of Yan is supported by the NSFC Grant No. 11771127.

\section{REFERENCES}

[1] M. S. Alber, R. Camassa, D. Holm and J. E. Marsden, The geometry of peaked solitons and billiard solutions of a class of integrable PDE's, Lett. Math. Phys., 32 (1994) 137-151.

[2] H. BAhouri, J. Y. Chemin And R. DAnChin, Fourier analysis and nonlinear Partial Differential Equations, Grundlehren der mathematischen Wissenschaften, vol. 343, Springer, Berlin (2011).

[3] L. BRANDOLESE, Local-in-space criteria for blowup in shallow water and dispersive rod equations, Comm. Math. Phys., 330 (2014) 401-414.

[4] L. BRAndolese And M. F. CORTEZ, Blowup issues for a class of nonlinear dispersive wave equations, J. Differential Equations, 256 (2014) 3981-3998.

[5] R. CAmassa And D. Holm, An integrable shallow water equation with peaked solitons, Phys. Rev. Lett., 71 (1993) 1661-1664.

[6] J. Y. Chemin, Localization in Fourier space and Navier-Stokes system, Phase Space Analysis of Partial Differential Equations, Proceedings 2004, CRM series, Pisa, 53-136.

[7] J. Y. Chemin, Perfect Incompressible Fluids, in: Oxford Lecture Series in Mathematics and its Applications, Vol. 14, The Clarendon Press, Oxford University Press, New York, (1998).

[8] A. Constantin, Nonliear Water Waves with Applications to Wave-Current Interactions and Tsunamis, volume 81 of CBMS-NSF Conference Series in Applied Mathematics, SIAM, Philadelphis, (2011).

[9] A. Constantin, Existence of permanent and breaking waves for a shallow water equation: a geometric approach, Ann. Inst. Fourier, 50 (2000) 321-362.

[10] A. Constantin And J. Escher, Analyticity of periodic traveling free surface water waves with vorticity Ann. of Math., 173 (2011) 559-568.

[11] A. COnStAntin And J. Escher, On the blow-up rate and the blow-up set of breaking waves for a shallow equation, Math. Z., 233 (2000) 75-91.

[12] A. Constantin And J. Escher, Wave breaking for nonlinear nonlocal shallow water equations, Acta Math., 181 (1998) 229-243.

[13] A. Constantin And J. Escher, Global existence and blow-up for a shallow water equation, Ann. Scuola Norm. Sup. Pisa, 26 (1988) 303-328.

[14] A. Constantin And D. LAnnes, The hydrodynamical relavance of the Camassa-Holm and Degasperis-Procesi equations, Arch. Rotional Mech. Anal, 192 (2009) 165-186. 
[15] R. DAnchin, A few remarks on the Camassa-Holm equation, Differential Integral Equations, 14 (2001) 953-988.

[16] R. Danchin, A note on well-posedness for Camassa-Holm equation, J. Differential Equations, 192 (2003) 429-444.

[17] R. DAnchin, Fourier Analysis Method for PDEs, Lecture Notes, vol. 14, (2005).

[18] H. A. ERbAy, S. ERbAY AND A. ERKIP, Derivation of the Camassa-Holm equations for elastic waves, Phys. Lett. A, 379 (2015) 956-961.

[19] M. FISHER AND J. SCHIFF, The Camassa Holm equation: conserved quantities and the initial value problem, Phys. Lett. A, 259 (1999) 371-376.

[20] L. L. FAN AND H. J. GAO, Local well-posedness and persistence properties for the variable depth KDV general equations in Besov space $B_{2,1}^{3 / 2}$, Differential and Integral Equations, 29 (2016) 241-268.

[21] L. L. FAN AND W. YAN, The Cauchy problem for shallow water waves of large amplitude in Besov space, J. Differential Equations, 267 (2019) 1705-1730.

[22] B. FuChSSTEIner AND A. S. FOKAS, Symplectic structures, their Bäklund transformation and hereditary symmetries, Physica D, 4 (1981) 47-66.

[23] Y. FU, G. GU, Y. LiU And Z. QU, On the Cauchy problem for the integrable Camassa-Holm type equation with cubic nonlinearity, J. Differential Equations, 255 (2013) 1905-1938

[24] Z. GAN, Y. HE AND L. MENG, Large time behavior and convergence for the Camassa-Holm equations with fractional Laplacian viscosity, Calc. Var., 57 (2018) doi:10.1007/s00526-018-1421-z.

[25] G. GUi AND Y. LiU, Global well-posedness and blow-up of solutions for the Camassa-Holm equations with fractional dissipation, Math. Z, 281 (2015) 993-1020.

[26] A. Himonas And G. Misiolek, Analyticity of the Cauchy problem for an integrable evolution equation, Math. Ann., 327 (2003) 575-584.

[27] A. Himonas, G. Misiolek, G. Ponce And Y. Zhou, Persistence properties and unique continuation of solutions of the Camassa-Holm equation, Comm. Math. Phys., 271 (2007) 511-522.

[28] J. Holmes And R. C. Thompson, Well-posedness and continuity properties of the FornbergWhitham equation in Besov spaces, J. Differential Equations, 263 (2017) 4355-4381.

[29] M. A. Johnson, Stability of small periodic waves in fractional KdV type equations, SIAM J. Math. Anal., 45 (2013) 3168-3193.

[30] R. S. Johnson, Camassa-Holm, Korteweg-de Vries and related models for water waves, J. Fluid Mech., 4 (2002) 63-82.

[31] T. KATO, Quasi-Linear Equations of Evolution, with Applications to Partical Differential Equations Spectral Theory and Differential Equation, Lecture Notes in Mathematics, 448 (1975) 25-70.

[32] X. LiU AND Z. Yin, Local well-posedness and stability of solitary waves for the two-component Dullin-Gottwald-Holm system, Nonlinear Analysis, 88 (2013) 1-15.

[33] C. Miao, J. Wu And Z. Zhang, Littlewood-Paley theory with applications to hydrodynamic equations (in Chinese), Volume 142 of Basic books on modern mathematics, Beijing Science Press, (2012).

[34] N. D. Mutlubas, On the Cauchy problem for the fractional Camassa-Holm equation, Monatshefte für Mathematik, 190 (2019) 755-768.

[35] Y. Mi AND C. MU, On the solutions of a model equation for shallow water waves of moderate amplitude, J. Differential Equations, 255 (2013) 2101-2129.

[36] L. Ni AND Y. ZHOU, Well-posedness and persistence properties for the Novikov equation, J. Differential Equations, 250 (2011) 3002-3021.

[37] J. A. PAVA, Stability properties of solitary waves for fractional KdV and BBM equations, Nonlinearity, 31 (2018) 920-956.

[38] K. YAn And Z. Y. Yin, Analytic solutions of the Cauchy problem for two-component shallow water systems, Math. Z, 269 (2011) 1113-1127.

[39] W. YAN, Y. Li AND Y. ZHANG, The Cauchy problem for the generalized Camassa-Holm equation in Besov space, J. Differential Equations, 256 (2014) 2876-2901. 
(Lili Fan) College of Mathematics and Information Science, Henan Normal University, XinxiANG 453007, CHINA

E-mail address: fanlily89@126.com

(Hongjun Gao) School of Mathematical Sciences, Institute of Mathematics, Nanjing Normal UNIVERSity, NANJING 210023, CHINA

E-mail address: gaohj@hotmail.com

(Junfang Wang) School of Mathematics and statistics, North China University of Water ReSources And Electric Power, Zhengzhou, Henan 450045, China

E-mail address: wang junfang18@ncwu.edu.cn

(Wei Yan) College of Mathematics and Information Science, Henan Normal University, XinXiANG 453007, CHINA

E-mail address: 011133 ahtu.edu.cn (Corresponding author) 